\title{
Scalable \& Resilient Vehicle-Centric Certificate Revocation List Distribution in Vehicular Communication Systems
}

\author{
Mohammad Khodaei, Member, IEEE, and Panos Papadimitratos, Fellow, IEEE
}

\begin{abstract}
In spite of progress in securing Vehicular Communication (VC) systems, there is no consensus on how to distribute Certificate Revocation Lists (CRLs). The main challenges lie exactly in (i) crafting an efficient and timely distribution of CRLs for numerous anonymous credentials, pseudonyms, (ii) maintaining strong privacy for vehicles prior to revocation events, even with honest-but-curious system entities, (iii) and catering to computation and communication constraints of on-board units with intermittent connectivity to the infrastructure. Relying on peers to distribute the CRLs is a double-edged sword: abusive peers could "pollute" the process, thus degrading the timely CRLs distribution. In this paper, we propose a vehicle-centric solution that addresses all these challenges and thus closes a gap in the literature. Our scheme radically reduces CRL distribution overhead: each vehicle receives CRLs corresponding only to its region of operation and its actual trip duration. Moreover, a "fingerprint" of CRL 'pieces' is attached to a subset of (verifiable) pseudonyms for fast CRL 'piece' validation (while mitigating resource depletion attacks abusing the CRL distribution). Our experimental evaluation shows that our scheme is efficient, scalable, dependable, and practical: with no more than $25 \mathrm{~KB} / \mathrm{s}$ of traffic load, the latest CRL can be delivered to $95 \%$ of the vehicles in a region $(15 \times 15 \mathrm{KM})$ within 15 s, i.e., more than 40 times faster than the state-of-the-art. Overall, our scheme is a comprehensive solution that complements standards and can catalyze the deployment of secure and privacy-protecting VC systems.
\end{abstract}

Index Terms-Vehicular Communications, VANETs, Vehicular PKI, Certificate Revocation, CRL Distribution, Security, Privacy, Efficiency

\section{INTRODUCTION}

Vehicle-to-Vehicle (V2V) and Vehicle-to-Infrastructure (V2I) communications seek to enhance transportation safety and efficiency with a gamut of applications, ranging from collision avoidance alerts to traffic conditions updates; moreover, they can integrate and enrich Location Based Services (LBSs) [1], |2] and vehicular social networks [3], and provide infotainment services. It has been well-understood that Vehicular Communication (VC) systems are vulnerable to attacks and that the privacy of their users is at stake. As a result, security and privacy solutions have been developed by standardization bodies (IEEE 1609.2 WG [4] and ETSI [1] [5], |6]), harmonization efforts (Car2Car Communication Consortium (C2C-CC) [7]), and projects (SeVeCom [8], PRESERVE [9], and CAMP [10]). A consensus towards using Public Key Cryptography ( $\overrightarrow{\mathrm{PKC}}$ ) to protect V2V/V2I (V2X) communication is reached: a set of Certification Authorities (CAs) constitutes the Vehicular Public-Key Infrastructure (VPKI), providing multiple anonymous credentials, termed pseudonyms, to legitimate vehicles. Vehicles switch from one pseudonym to a non-previously used one towards unlinkability of digitally signed messages, and improved sender privacy for V2V/V2I messages. Pseudonymity is conditional in the sense that the corresponding long-term vehicle identity (Long Term Certificate (LTC)) can be retrieved by the VPKI entities if deviating from system policies.

In fact, vehicles can be compromised or faulty and disseminate erroneous information across the V2X network [11], [12]. They should be held accountable for such actions and credentials (their LTCs and their pseudonyms) can be revoked. To efficiently revoke a set of pseudonyms, one can disclose a single entry for all (revoked) pseudonyms of the vehicle [13], [14], [15], [16]. However, upon a revocation event, all non-revoked (but expired) pseudonyms belonging to the "misbehaving" vehicle would also be linked. Linking pseudonyms with lifetimes prior to a revocation event implies that all the corresponding digitally signed messages will be trivially linked. Even if revocation is justified, this does not imply that a user "deserves" to abolish privacy prior to the revocation event. Avoiding such a situation, i.e., achieving what is termed in the literature as perfectforward-privacy [17], can be guaranteed if the VPKI entities are fully-trustworthy [18]. However, we need to guarantee strong user privacy even in the presence of honest-but-curious VPKI entity; recent revelations of mass surveillance show that assuming service providers are fully-trustworthy is no longer a viable approach.

A main concern, relevant to all proposals in the literature [15], [16], [18], [19], [20], [21] is efficiency and scalability, essentially low communication and computation overhead even as system dimension grows. Consider first typical operational constraints: the average daily commute time is less than an hour (on average 29.2 miles and 46 minutes per day) [10], [22], [23] while the latencies for the dissemination of a full Certificate Revocation List (CRL) can exceed the actual trip duration [24]. One can compress CRL using a Bloom Filter (BF) [12], |25], [26]; however, the size of a CRL grows linearly with the number of revoked pseudonyms, thus necessitates larger BFs. More so, a sizable portion of the CRL information is irrelevant to a receiving vehicle and can be left unused. This, at the system level, constitutes waste 
of computation, communication (bandwidth), and storage resources. In turn, it leads to higher latency for all vehicles to reconstruct the CRL, i.e., a degradation of timely distribution.

Alternatively, vehicles can only validate revocation status of (their neighbors') pseudonyms via an Online Certificate Status Protocol (OCSP). Even if a VPKI can comfortably handle such a demanding load [27], OCSP cannot be used as a standalone solution in VC systems: it requires continuous connectivity and significant bandwidth dedicated to revocation traffic, thus impractical due to the network volatility and scale [12]. Moreover, what would be the course of action if the VPKI were not reachable for other reasons, e.g., during a Denial of Service (DoS) attack? So, the challenge is how can one distribute the most relevant revocation information to a given vehicle, per trip, and ensure timely revocation even without uninterrupted connectivity to the VPKI?

The computation overhead for the verification of the CRL could interfere with safety- and time-critical operations especially if one considers typical VC rates of 10 safety beacons per second, and thus processing of possibly hundreds of messages from neighboring vehicles per second. Simply put, with existing computation and communication overhead and the time-critical nature of safety applications, minimizing the overhead for CRL verification and distribution is paramount.

From a different viewpoint, we need to allocate as little bandwidth as possible for the CRL distribution in order not to interfere with safety-critical operations or enable an attacker to broadcast a fake CRL at a high rate. However, this should be hand in hand with timely CRL distribution. This can be achieved with the use of Roadside Units (RSUs) |19|; however, dense deployment of RSUs in a large-scale environment is costly. If the deployment is sparse, a significant delay could be introduced. Alternatively, the CRL can be distributed in a peer-to-peer, epidemic manner [15], [16], [18]. This is a double-edged sword: abusive peers, seeking to compromise the trustworthiness of the system, could pollute the CRL distribution and mount a clogging DoS attack. Such an attack relates other content dissemination, e.g., vehicular social networks [3], yet it is critical to mitigate it for CRL distribution: delaying or preventing legitimate users from obtaining the most up-to-date CRL pieces would result in prolonging the operation of a malicious compromised vehicle in the system.

Moreover, a vehicle could be wrongly identified as a misbehaving entity, e.g., a false positive in an eventbased misbehavior detection [25], [28], or a vehicle sensor malfunction, e.g., a communication breakdown or a problem in a safety-critical system (e.g., steering or braking [29]). Thus, the LTC as well as all pseudonyms of the "misbehaving" entity would be revoked, with the revocation information to be distributed/made available to all vehicles in the network. This raises two challenges: misbehavior could be falsely identified, or the sensor malfunctioning could be transient, thus distributing "imprecise" revocation information, likely in high volumes. The effect would be waste of communication (bandwidth) and higher convergence time to reconstruct the CRL, i.e., degradation of timely CRL distribution. Repairing a faulty sensor or patching the vehicle software or resolving the erroneous eviction implies the vehicle can rejoin the system as legitimate participant. This means the vehicle needs to obtain a fresh batch of pseudonyms, e.g., for another three years [10], i.e., imposing unnecessary workload on the VPKI entities. Therefore, a flexible design with easily reversible revocation status that allows to temporarily evict a misbehaving or malfunctioning vehicle from the system until the issue is resolved would address this challenge. By the same token, we need to ensure that such temporal eviction of a vehicle does not harm user privacy; in other words, exactly because such a flexible design allows resolution and temporal eviction, a single VPKI entity should not be able to link the corresponding (successive) pseudonyms of the same vehicle, upon rejoining the system.

Furthermore, the VPKI system must narrow down the vulnerability window, i.e., the interval between a revocation event/incident until all vehicles successfully obtain the latest revocation information. Thus, if a new revocation event happens when the base-CRL has already been distributed, the VPKI entities should release $\Delta$-CRLs in order to close down the vulnerability window. However, frequent distribution of such revocation information could affect the performance of VC systems. More important, abusive peers could mount a clogging DoS attack on the $\Delta$-CRLs distribution. A lightweight, i.e., incurring low computation and communication overhead, and resilient mechanism for distributing $\Delta$-CRLs would address this challenge.

Despite the plethora of research efforts, none addresses all challenges at hand. In this paper, we show how to efficiently revoke a very large volume of pseudonyms while providing strong user privacy protection, even in the presence of honestbut-curious VPKI entities. Our system effectively, resiliently, and in a timely manner disseminate the authentic CRL throughout a large-scale (multi-domain) VC system. Moreover, we ensure that the CRL distribution incurs low overhead and prevents abuse of the distribution mechanism. Furthermore, our flexible design allows to temporarily evict a vehicle from the system without compromising user privacy. At the same time, it facilitates rejoining the system as a legitimate participant upon resolving the issue without imposing unnecessary workload on the VPKI entities, by frequently refilling pseudonyms pool, and, most important, shields the system from clogging DoS attacks leveraging the CRL and $\triangle-C R L$ distribution.

Contributions: Our comprehensive security and privacypreserving solution systematically addresses all key aspects of CRL-based revocation, i.e., security, privacy, and efficiency. This is based on few simple yet powerful, as it turns out, ideas. We propose making the CRL acquisition process vehicle-centric: each vehicle only receives the pieces of CRLs corresponding to its targeted region and its actual trip duration, i.e., obtaining only region- and time-relevant revocation information. Moreover, randomly chosen pseudonyms issued by the VPKI are selected to piggyback a notification about new CRL-update events and an authenticator for efficiently validating pieces of the latest CRL; in other words, validation of the CRL pieces almost for free. These novel features dramatically reduce the CRL size and CRL validation overhead, while they significantly increase its resiliency against resource depletion attacks. Moreover, we propose a secure, efficient, and resilient distribution mechanism for $\Delta$-CRL updates to narrow down the vulnerability window. Furthermore, our scheme facilitates eviction of a "misbehaving" vehicle temporarily, i.e., for an interval until the issue is resolved.

In the rest of the paper, we critically survey the literature 
(Sec. 2) and explain the system model (Sec. 3). We present system design (Sec. 4), followed by qualitative and quantitative analysis (Sec. 5). We then conclude the paper (Sec. 6).

\section{Related Work}

The need to evict misbehaving or compromised [11] vehicles from a VC system is commonly accepted, because such vehicles can threaten the safety of vehicles and users and degrade transportation efficiency. CRL distribution is of central importance and it is the final and definitive line of defense [1], [4], [8], [8], [25], [30]: only the VPKI can "ultimately" revoke a vehicle by including its unexpired certificates' serial numbers in a CRL.

The literature proposes distribution of the CRL via RSUs [19] and car-to-car epidemic communication [15], [16], [18], with enhancements on the distribution of pieces [20], [21] evaluated in [31]. A naïve solution would be to digitally sign the entire CRL and broadcast it; however, it imposes difficulties in downloading a large CRL file and exchanging it over short contact period (with an RSU or a peer). Splitting the digitally signed CRL into multiple pieces is vulnerable to pollution attacks: in the absence of fine-grained authentication, per CRL piece, an adversary can delay or even prevent reception by injecting fake pieces. Thus, the straightforward solution is to have the VPKI prepare the CRL, split it into multiple pieces, sign each piece, and distribute all of them across the VC system. RSUs can broadcast CRL pieces randomly or in a round-robin fashion [19], and vehicles can relay pieces until all vehicles receive all pieces necessary to reconstruct the CRL [15]. Erasure codes can be used to enhance the fault-tolerance of the CRL piece distribution in the highly volatile VC environment [19], [32].

Signing each CRL piece so that it is self-verifiable, incurs significant computation overhead, which grows linearly with the number of CRL pieces, both for the VPKI and for the receiving vehicles. Furthermore, an attacker could aggressively forge CRL pieces for a Distributed DoS (DDoS) attack leveraging signature verification delays [33] that can prevent vehicles from obtaining the genuine CRL pieces. A "precode-and-hash" scheme [34] proposes to calculate a hash value of each pre-coded piece, sign it, and disseminate it with higher priority. Each relaying node can apply a different precode to the original CRL and act as a secondary source. However, by applying different encodings to the original CRL file, another receiver cannot reconstruct the entire CRL from the pieces, encoded differently by various relaying nodes. To mitigate pollution and DoS attacks, we propose to piggyback a fingerprint (a BF [35], [36]) for CRL pieces into a subset of pseudonyms to validating CRL pieces "for free".

To efficiently revoke an ensemble of pseudonyms, one can enable revocation of multiple pseudonyms with a single CRL entry, to reduce the CRL size, e.g., [15], [16], [37]. Despite a huge reduction in size, such schemes do not provide perfect-forward-privacy [17]: upon a revocation event and CRL release, all the "non-revoked" but previously expired pseudonyms belonging to the evicted entity would be linked as well. Although forward-privacy can be achieved by leveraging a hash chain [18], the pseudonyms' issuer can trivially link all pseudonyms belonging to a vehicle, and thus the pseudonymously authenticated messages [38],
[39], [40], towards tracking it for the entire duration of its presence in the system [13], [14], [15], [16], [18]. More precisely, the CA specifies a "time interval" so that each vehicle receives $\mathbb{D}$ pseudonyms during the pseudonym acquisition process $[18]$. As a result, for each batch of revoked pseudonyms, a single key is disclosed. But, upon a revocation event, all pseudonyms within an interval are linked, because one can decrypt all pseudonym serial numbers; thus, no perfect-forward-privacy is achieved for that period. On the contrary, in our scheme, upon a revocation event and CRL release, it is infeasible to link the previously non-revoked (but expired) pseudonyms belonging to a misbehaving vehicle. This is so due to the utilization of a hash chain during the pseudonym issuance process, thus achieving perfect-forwardprivacy [41].

Compressing CRLs using a BF was proposed for compact storage of revocation entries [25], or to efficiently distribute them across the network [12], [25], [26]. However, the challenge is twofold: scalability and efficiency. Their CRL size still grows linearly with the number of revoked pseudonyms, while a substantial portion of the compressed CRL can be irrelevant to a receiving vehicle and be left unused. Moreover, compressing CRLs using a BF does not necessarily reduce the size of a CRL as vehicles can be provided with possibly hundreds of pseudonyms [4], [41], [42]. Unlike such schemes [12], [25], [26], we do not compress the CRL: our scheme disseminates only triprelevant revocation information to vehicles and it utilizes a $\mathrm{BF}$ to provide a condensed authenticator for the CRL pieces. Our scheme leverages and enhances the functionality of the state-of-the-art VPKI system [43] towards efficiently revoking a batch of pseudonyms without compromising user privacy backwards: upon a revocation event, all pseudonyms prior to the revocation event remain unlinkable.

Alternatively, vehicles could validate pseudonym status information through OCSP. But, due to intermittent network connectivity, significant usage of the bandwidth by time- and safety-critical operations, and substantial overhead for the VPKI (if it is reachable), OCSP cannot be used as a standalone solution [12]. A hybrid solution could rely on distributing certificate status information to other mobile nodes [44], [45]; however, the system would be subject to the reachability (of sufficiently many cooperative) and the trustworthiness of such nodes. In our scheme, we ensure that the latest CRL is efficiently, effectively, and timely distributed among all vehicles without any assumption on persistent reachability and trustworthiness of specific mobile nodes.

Temporal eviction of a misbehaving or malfunctioning vehicle from the VC system has received limited attention. There are several situations that a vehicle should be temporarily evicted from the system until the issue is resolved and the vehicle can rejoin the system, e.g., in case a malfunctioning sensor disseminating false information. Security Credential Management System (SCMS) [10], [46] supports only permanent eviction of a misbehaving vehicle by including a linkage seed into a CRL. Towards temporal revocation of credentials, [47], [48] propose a linkage hook between any linkage seed and the corresponding prelinkage values in the original SCMS design. Thus, in order to temporarily revoke the credentials, the linkage hook is disclosed (instead of the linkage seed, used for permanently 
revoking the credentials). Temporal eviction of a subset of the certificates requires additional layers to be added to the tree. However, the disclosure of linkage hooks would trivially link all pseudonyms inside a given subtree. Our scheme facilitates eviction of a misbehaving vehicle temporarily, i.e., for a fine-grained interval, until the issue is resolved without compromising user privacy (prior to the revocation event).

SCMS [10], [46] issues pseudonyms with the help of two Linkage Authorities (LAs): a batch of 20-40 pseudonyms, valid for a week, are issued for each vehicle without having a single VPKI entity able to link them. In case of revocation, a single entry is disclosed to invalidate the batch of revoked pseudonyms. As a result of this binding, the size of a CRL linearly grows with the number of compromised vehicles (and not with the number of revoked pseudonyms). However, due to large number of pseudonyms carried by each vehicle for a long period, e.g., 3 years [10] or 25 years [46] the size of a CRL could be huge [42]. The VPKI [10], [46] could provision vehicles for a long period, e.g., 25 years worth of pseudonyms, with a decryption key for, e.g., a weekly batch of pseudonyms, delivered periodically [42], [46], [49]. This would eliminate the need for frequently recurring bidirectional connectivity to the VPKI to obtain pseudonyms. To evict a vehicle, the VPKI can stop delivering the corresponding decryption key to the vehicle Hardware Security Module (HSM). Still, it is imperative to distribute the CRL and cover the (week long) period and the corresponding revoked pseudonyms. Furthermore, having released a CRL towards the end of a week, signed messages with the private keys corresponding to the recently revoked pseudonyms (included in the CRL) can be linked, i.e., achieving no perfectforward-privacy for that period [24].

\section{Model AND RequiRements}

\subsection{System Model and Assumptions}

A VPKI consists of a set of Certification Authorities (CAs) with distinct roles: the Root CA (RCA), the highest-level authority, certifies other lower-level authorities; the Long Term CA (LTCA) is responsible for the vehicle registration and the Long Term Certificate (LTC) issuance, and the Pseudonym CA (PCA) issues pseudonyms for the registered vehicles. Pseudonyms have a lifetime (a validity period), typically ranging from minutes to hours; in principle, the shorter the pseudonym lifetime is, the higher the unlinkability and thus the higher privacy protection can be achieved. We assume that each vehicle is registered only with its Home-LTCA (H-LTCA), the policy decision and enforcement point, reachable by the registered vehicles. Without loss of generality, a domain can be defined as a set of vehicles in a region, registered with the H-LTCA, subject to the same administrative regulations and policies [50]. There can be several PCAs, each active in one or more domains. Trust between two domains can be established with the help of the RCA, or through cross certification.

All vehicles (On-Board Units (OBUs)) registered in the system are provided with HSMs, ensuring that private keys never leave the HSM. Moreover, we assume that there is a misbehavior detection system, e.g., [51], that triggers the revocation. The Resolution Authority (RA) can initiate a process to resolve and revoke all pseudonyms of a misbehaving vehicle: it interacts with the corresponding PCAs and LTCA (a detailed protocol description in [27], [43], [52]) to resolve and revoke all credentials issued for a misbehaving vehicle. Consequently, the misbehaving vehicle can no longer obtain credentials from the VPKI. The VPKI is responsible for distributing the CRLs and notifying all legitimate entities about the revocation; this implies a new CRL-update event.

\subsection{Adversarial Model and Requirements}

We extend the general adversary model in secure vehicular communications [53] to include VPKI entities that are honestbut-curious, i.e., entities complying with security protocols and policies, but motivated to profile users. In a VC environment, internal adversaries, i.e., malicious, compromised, or non-cooperative clients, and external adversaries, i.e., unauthorized entities, raise four challenges. More specifically in the context of this work, adversaries can try to (i) exclude revoked pseudonym serial numbers from a CRL, (ii) add valid pseudonyms by forging a fake CRL (piece), or (iii) prevent legitimate entities from obtaining genuine and the most up-to-date CRL (pieces), or delay the CRL distribution by replaying old, spreading fake CRL (pieces), or performing a DoS attack. This allows wrong-doers to remain operational in the VC system using their current revoked pseudonym sets. Moreover, they might be simply non-cooperative or malicious, tempted to prevent other vehicles from receiving a notification on a new CRL-update event, thus preventing them from requesting to download the CRLs. Lastly, (iv) VPKI entities (in collusion with vehicle communication observers) could potentially link messages signed under (nonrevoked but expired) pseudonyms prior to the revocation events, e.g., inferring sensitive information from the CRLs towards linking pseudonyms, and thus tracking vehicles backwards. The PCAs operating in a domain (or across domains) could also collude, i.e., share information that each of them individually has, to harm user privacy.

Security and privacy requirements for V2X communications have been specified in the literature [53], and additional requirements for VPKI entities in [43]. The security and privacy requirements for the CRL distribution are: finegrained authentication, integrity, and non-repudiation, unlinkability (perfect-forward-privacy), availability, efficiency, and explicit and/or implicit notification on revocation events [41]. Beyond these requirements, the revocation mechanism should be flexible and provide the option for easily reversible certificate status. More precisely, the system should facilitate temporal eviction of a misbehaving or malfunctioning vehicle from the system. By the same token, the revocation mechanism should allow reinstating an entity previously revoked upon resolving a misbehaving or malfunctioning issue that led to the revocation; the temporarily-evicted entity could rejoin the system and continue operating in the VC system. Even if a vehicle is preloaded with pseudonyms for a longer period, e.g., a year, it should be able to leverage the already obtained credentials upon rejoining the system. 


\section{DESIGN}

\subsection{Motivation and Overview}

Preliminary assumptions: We leverage a state-of-the-art VPKI system [43], [54], [55] that provides pseudonyms in an on-demand fashion: each vehicle "decides" when to trigger the pseudonym acquisition process based on various factors [56], [57. Such a scheme requires sparse connectivity to the VPKI, but it facilitates an OBU to be preloaded with pseudonyms proactively, covering a longer period, e.g., a week or a month, should the connectivity be expected heavily intermittent. The efficiency, scalability and robustness of the VPKI system is systematically investigated [43], [54], [55], [56] with the VPKI handles a large workload. Moreover, it enhances user privacy, notably preventing linking pseudonyms based on timing information [38] (the instance of issuance and the pseudonym lifetime) as well as offers strong user privacy protection even in the presence of honest-but-curious VPKI entities. More precisely, a universally fixed interval, $\Gamma$, is specified by the H-LTCA and all pseudonyms in that domain are issued with the lifetime $\left(\tau_{P}\right)$ aligned with the VPKI clock. Vehicles obtain pseudonyms on-the-fly as they operate, and the number of pseudonyms in a request is $\frac{\Gamma}{\tau_{p}}$, i.e., no prior calculation needed. As a result of this policy, at any point in time, all vehicles pseudonyms are indistinguishable based on issuance time thanks to this time alignment, i.e., eliminating any distinction among pseudonym sets of different vehicles, thus enhancing user privacy. We leverage and enhance the functionality of this VPKI system; in particular, our solution necessitates two modifications during pseudonym acquisition process, notably (i) implicitly binding pseudonyms issued to a given requester per $\Gamma$, and (ii) integrating a fingerprint into a subset of the pseudonyms for efficient CRL validation (detailed description in [41]).

High-level overview: The default policy is to distribute all revocation information to all vehicles. Nonetheless, this approach ignores the locality, the temporal nature of pseudonyms, and other constraints, e.g., the average daily commute time. Locality could be geographical, i.e., credentials relative to the corresponding region, and temporal, i.e., relevance to the lifetime of pseudonyms with respect to the trip duration of a vehicle. To efficiently, effectively, and timely distribute the CRLs across the V2X network, we propose making the CRL acquisition process vehicle-centric, i.e., through a contentbased and context-sensitive "publish-subscribe" scheme [58], [59].

By starting a new trip, each vehicle only subscribes to receive the pieces of CRLs, i.e., the content, corresponding to its actual trip duration and its targeted region, i.e., the context. To reap the benefits of the ephemeral nature pseudonyms and the timely-aligned pseudonym provisioning policy, towards an effective, efficient, and scalable CRL distribution, a fixed interval, $\Gamma_{C R L}$, is predetermined by the PCAs in the domain. They publicize revoked pseudonyms whose lifetimes fall within $\Gamma_{C R L}$, i.e., distributing only the serial number of these pseudonyms rather than publishing the entire CRL. Note that $\Gamma$, the universally fixed interval to obtain pseudonyms [43], and $\Gamma_{C R L}$ are not necessarily aligned due to the unpredictable nature of revocation events.

When a vehicle reliably connects to the VPKI, it can obtain the "necessary" CRL pieces corresponding to its trip duration during the pseudonym acquisition phase. However,

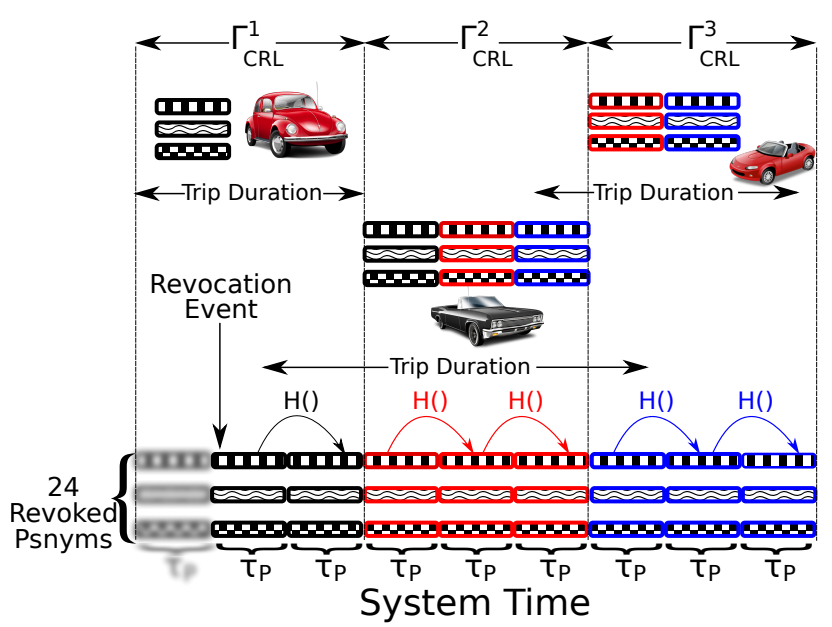

Fig. 1. A vehicle-centric approach: each vehicle only subscribes for pieces of CRLs corresponding to its trip duration.

if reliable connectivity is not guaranteed, or if a vehicle obtained (possibly preloaded with enough) pseudonyms in advance, or a new revocation event happens, one can be notified about a new CRL-update (revocation) event: a signed fingerprint (a Bloom Filter (BF) [35], [36]) of CRL pieces is broadcasted by RSUs; furthermore, it is appended in a subset of recently issued pseudonyms for a subset of vehicles (termed fingerprint-carrier nodes); these pseudonyms are attached to as typically all Cooperative Awareness Messages (CAMs). This essentially piggybacks a notification about the latest CRL-update event and an authenticator for validating CRL pieces. This provides CRL validation for free in terms of computational overhead: pseudonyms are readily validated by the receiving vehicles since each vehicle verifies the signature on a pseudonym before validating the content of a CAM, i.e., the verification of CRL pieces does not incur extra computation overhead. This eliminates the need for signature verification, but a BF membership test, for each CRL piece as the fingerprint is signed by the PCA.

Our scheme does not require prior knowledge on trip duration to obtain CRLs, i.e., a vehicle can be oblivious to the trip duration. In fact, such information would not be relevant to the CRL dissemination: due to the unpredictable nature of revocation events, the PCAs disseminate at each point revoked pseudonyms whose lifetimes fall within a $\Gamma_{C R L}$ interval. In other words, even if a vehicle knows the trip duration, it will not receive revocation information regarding the far future. In contrast, the revocation information is progressively distributed among the vehicles. The reason is twofold: first, trivially, some revocation events are not yet scheduled; receiving CRLs within a near time horizon is more likely to include the latest revocation information. Moreover, upon resolving the issue that led to the revocation of a malfunctioning or misbehaving entity before the next update of the CRL, the corresponding credentials should not be included in the CRL, i.e., achieving reversible revocation status. The percentage of the information that is relevant at a given point in time, and is included in the CRL, is a function of pseudonym lifetime $\left(\tau_{p}\right)$, and the size of the $\Gamma_{C R L}$ interval. More precisely, the shorter the pseudonym lifetime $\left(\tau_{p}\right)$ and the longer the $\Gamma_{C R L}$ intervals are, the higher the number of revocation entries is, i.e., the larger the CRL size. 


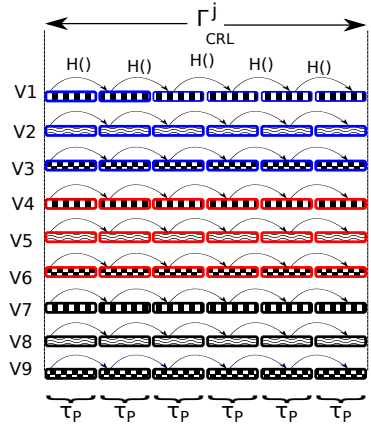

(a) Revoked pseudonyms.

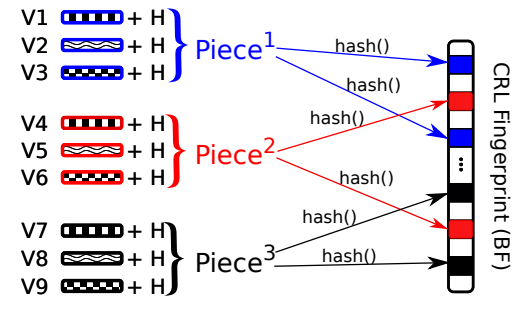

(b) A CRL fingerprint construction.
Fig. 2. (a) Revoked pseudonyms in a $\Gamma_{C R L}^{j}$. (b) Fingerprint construction.

Fig. 11illustrates an example of 24 revoked pseudonyms to be distributed. A vehicle traveling within $\Gamma_{C R L}^{1}$ would possibly only face revoked pseudonyms with a lifetime falling in that interval, 6 pseudonyms, shown in black, instead of all 24 entries (the blurred pseudonyms are expired, thus not included in the CRL). These 6 revoked pseudonyms within $\Gamma_{C R L}^{1}$ can be implicitly bound without compromising their unlinkability prior to the revocation event, in a way that one can simply derive subsequent pseudonyms from an anchor (the blurred pseudonyms are non-revoked but expired and they cannot be linked to the revoked ones). Thus, in this example, distributing 3 entries for that vehicle is sufficient. Another vehicle, however, traveling for a longer duration, from the middle of $\Gamma_{C R L}^{1}$ till the beginning of $\Gamma_{C R L}^{3}$, would need to be provided with all 24 revocation entries, i.e., requiring 9 entries to derive all 24 revoked pseudonyms.

Fig. 2 shows how the PCA condenses the revocation information corresponding to nine compromised vehicles, each having 6 pseudonyms within a $\Gamma_{C R L}^{j}$ interval. The PCA utilizes the hash chain value for each set of revoked pseudonyms, generated during the pseudonym acquisition process [41]. Exactly because downloading a large CRL file is challenging, with vehicles and RSUs disseminating or exchanging the CRL over short contact (vehicle-to-vehicle or vehicle-to-RSU) periods, the PCA splits a large CRL into several pieces constructed by including the serial number of the first revoked pseudonym along with the corresponding complementary ${ }^{1}$ information. In order to ensure the authenticity and integrity of each CRL piece, the trivial solution is to sign each piece. However, this would incur significant computation and communication overhead, which grows linearly with the number of CRL pieces, both for the VPKI and for the receiving vehicles.

For efficient validation of CRL pieces, the PCA condenses authentication information for all the CRL pieces by constructing a probabilistic data structure, i.e., a Bloom Filter (BF). This condensed fingerprint is signed by the PCA and periodically broadcasted by RSUs; moreover, it is also integrated into a subset of recently issued pseudonyms, which are broadcasted along with CAMs. This notifies other vehicles about a new revocation event (and thus an updated

1. The complementary information is constructed by the PCA during pseudonym acquisition process: the PCA implicitly correlates a batch of pseudonyms belonging to each requester. This essentially enables efficient distribution of the CRL because the PCA only needs to include one entry per batch of pseudonyms without compromising their unlinkability. We refer interested readers to our earlier work 41 . for the detailed protocol description.

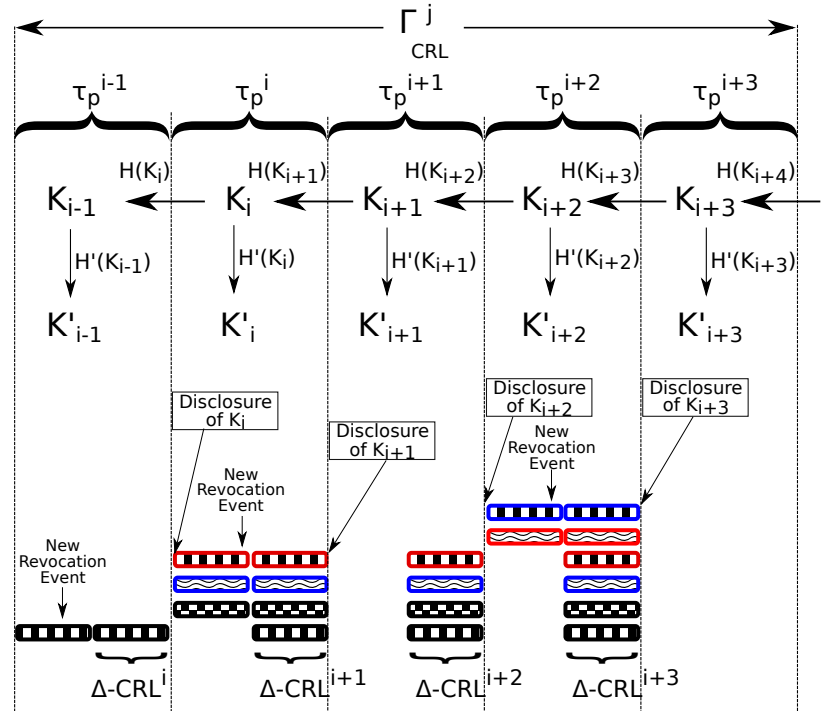

Fig. 3. Vehicle-centric $\Delta-C R L$ distribution.

CRL) as well as facilitates very fast validation of CRL pieces. This authenticator (or notification) is a condensed authenticator for the CRL pieces, and it can be broadcasted within a region very fast. Pseudonyms by default have to be authenticated by any vehicle. This implies validation of CRL pieces almost for free. Note that the PCA does not compress the CRL using a BF; rather, it utilizes a BF for efficiently validating CRL pieces. In fact, our vehicle-centric scheme does not need to compress CRLs because it effectively and progressively distributes revocation information corresponding to the actual vehicle trip duration.

$\Delta$-CRL updates: Each $\Gamma_{C R L}$ interval could potentially have a base-CRL, including non-expired revoked pseudonyms whose lifetimes fall within that $\Gamma_{C R L}$ interval. If new revocation event happen within a given $\Gamma_{C R L}$ interval, the PCA releases $\Delta$-CRLs to narrow down the vulnerability window, i.e., the interval between a revocation event until the time all vehicles successfully obtain the latest revocation information. $\Delta$-CRLs disseminate the revoked but not-yetexpired pseudonyms that are not included in the base-CRL. To ensure the authenticity of each $\Delta$-CRL (piece), a trivial solution would be for the PCA to either sign each piece, i.e., the baseline scheme, or construct another fingerprint and sign it, i.e., similar to vehicle-centric CRL distribution. However, due to unpredictable nature and potentially multiplicity of the revocation events, and more important, resilience considerations, i.e., performing a signature flooding attack [33| by broadcasting fake pieces, such solutions are not desirable.

Fig. 3 shows how the latest revocation information is disseminated within a $\Gamma_{C R L}^{j}$ interval. The PCA creates a hash chain, $N$ times, for each $\Gamma_{C R L}^{j}$, where $N$ is $\frac{\Gamma_{C R L}}{\tau_{p}}$ and it assigns the hash values sequentially to the time intervals, i.e., one key per $\tau_{P}$. Each hash value represents a cryptographic key to compute a Message Authentication Code (MAC), similar to [60], for all the $\Delta$-CRL pieces for that interval. The hash anchor is signed by the PCA and is included in the base-CRL pieces. Each $\Delta$-CRL piece contains the most recent revocation information, not included in the base-CRL, and a MAC of that piece. The PCA then reveals these hash values in the reverse order of generation. At every pseudonym 
transition proces $\mathrm{1}^{2}$, the PCA discloses the most recent oneway hash value, i.e., the key to the MAC, that it can disclose.

As Fig. 3 shows, a new revocation event happened in interval $i-1$, e.g., a vehicle was compromised and it disseminates erroneous information across the network. Thus, all credentials corresponding to that vehicle should be revoked. The PCA constructs a $\Delta$-CRL by including the serial number of the revoked pseudonym to be used during interval $i$. The PCA distributes the $\Delta$-CRL pieces to be used during interval $i$ during the interval $i-1$. Upon a pseudonym transition, the PCA discloses $K_{i}$, the cryptographic key to compute the MAC of the $\Delta$-CRL pieces. In the next interval $i$, there is a new revocation event and three more vehicles are compromised. The PCA constructs a $\Delta$-CRL piece, corresponding to interval $i+1$, and includes four entries (including the revoked one from the previous event). Again, upon a pseudonym transition, the PCA reveals $K_{i+1}$ to authenticate the $\Delta$-CRL pieces corresponding to the interval $i+1$. Note that in order to facilitate a fine-grained and reversible pseudonym revocation status, the PCA does not disclose the complementary information for the revoked pseudonyms in each $\Delta$-CRL piece (unlike the base-CRLs). Rather, it progressively and accumulatively distributes the revocation information in each pseudonym interval. This way, upon resolving the issues that led to the revocation of a misbehaving or malfunctioning vehicle, the PCA can easily stop including the serial number of the pseudonyms, belonging to that vehicle, into the $\Delta$-CRLs.

Note that within each time interval $\left(\tau_{P}^{i}\right)$, the PCA distributes $\Delta$-CRL pieces including the serial number of pseudonyms, whose lifetimes fall within the next time interval $\left(\tau_{P}^{i+1}\right)$. However, vehicles cannot authenticate the $\Delta$-CRL pieces until the PCA discloses the cryptographic key for that interval. Since vehicles know the schedule for disclosing the secret key (every $\tau_{P}$ ) and they are loosely synchronized with the VPKI clock, they wait for the delayed disclosure of keys at every pseudonym transition ${ }^{3}$ Upon receiving the secret key, vehicles can validate the buffered $\Delta$-CRL piece(s). However, after the disclosure of the secret key for an interval, vehicles do not accept any $\Delta$-CRL piece because such pieces could have been manipulated, i.e., forged by an adversary with the knowledge of the recently released key. Note that RSUs push $\Delta$-CRLs as well as the corresponding secret keys throughout the entire VC systems; as a result, the distribution of $\Delta$-CRLs in this way does not affect (change) the distribution of base-CRLs.

This flexible design allows to temporarily evict an entity from the system: during each $\tau_{P}$, the PCA only distributes serial numbers of revoked pseudonyms, whose validity intervals fall within the successive $\tau_{P}$. Upon resolving the misbehavior or malfunctioning, the PCA is informed (by the RA) and it does not include the serial number of the previously revoked entity into the CRLs or $\Delta$-CRLs. Thus, our vehicle-centric scheme provides a flexible, reversible,

2. Pseudonym transition is the process of switching from one pseudonym (and the corresponding private key) to another one (ideally, non-previously used) to ensure message unlinkability.

3. Note that we assume the VPKI issues time-aligned pseudonyms, with non-overlapping interval, for all vehicles; thus, all vehicles know the exact time key disclosure by the PCA and they change their pseudonyms at the same time [43].
TABLE 1

Notation Used in the Protocols.

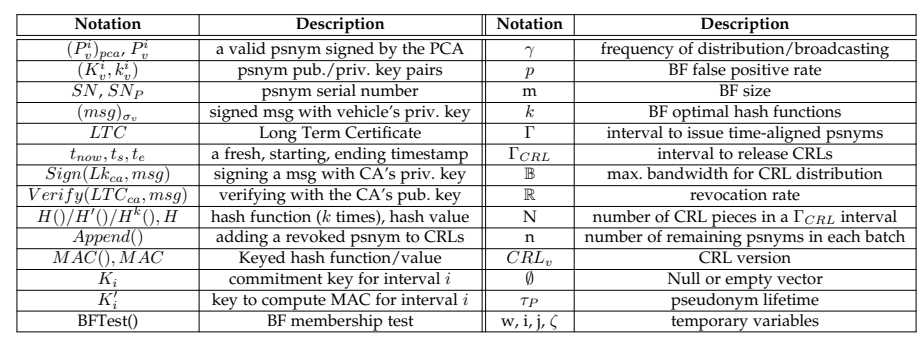

and fine-grained revocation management towards evicting a malfunctioning or misbehaving vehicle for an interval until the issue is resolved. Moreover, this procedure does not harm user privacy: the PCA cannot identify the actual identity of a misbehaving vehicle; also, upon resolving the issue, the PCA, as the issuer, can only link successive pseudonyms until the end of $\Gamma$; thus, user privacy is not degraded. Further discussion on security and privacy in Sec. 5

\subsection{Security Protocols}

In a nutshell, the PCAs operating in a domain construct the CRLs [41] and $\Delta$-CRLs by sorting the revoked pseudonyms based on their validity periods in a $\Gamma_{C R L}$ interval and push them to the RSUs. For ease of exposition, we assume there is one PCA, even though the extension with multiple PCAs within a given domain is straightforward. RSUs and fingerprint-carrier peers publish the CRL-update notification and the CRL pieces (Sec. 4.2.1). Moreover, RSUs push $\triangle$-CRLs and the MAC secret keys throughout the entire VC systems (Protocol 1 in Sec. 4.2.2. Upon receiving a new revocation event, each vehicle broadcasts a query to its neighbors to fetch the (missing) pieces of the CRL/ $\Delta$-CRL, e.g., similarly to [61], corresponding to its actual trip duration (Sec. 4.2.3). Finally, it parses recovered CRL pieces and stores them locally (Protocol[2] in Sec.4.2.4). Due to space limitations, we refer readers to our prior work [41] for the pseudonym acquisition process and CRL construction protocols. The notation is given in Table 1

\subsubsection{Publishing the CRL}

Each RSU continuously broadcasts the signed fingerprint of CRL pieces, to notify vehicles in a region about any new revocation event. The transmission rate of the signed fingerprint corresponding to the current $\Gamma_{C R L}^{i}$ can gradually decrease towards the end of $\Gamma_{C R L}^{i}$; instead, the transmission rate of the signed fingerprint for $\Gamma_{C R L}^{i+1}$ can moderately increase. This "ensures" that all legitimate vehicles are notified about a new revocation event, thus being capable to request and efficiently validate CRL pieces. Upon reception and validation of a query, an RSU commences transmission across the wireless data link with a low-rate transmission (without any acknowledgment from peers). Upon receiving an authentic query for the missing CRL pieces by a neighboring vehicle, a vehicle searches its local repository and randomly chooses one of the requested pieces and broadcasts it. A detailed protocol description in [41]. 


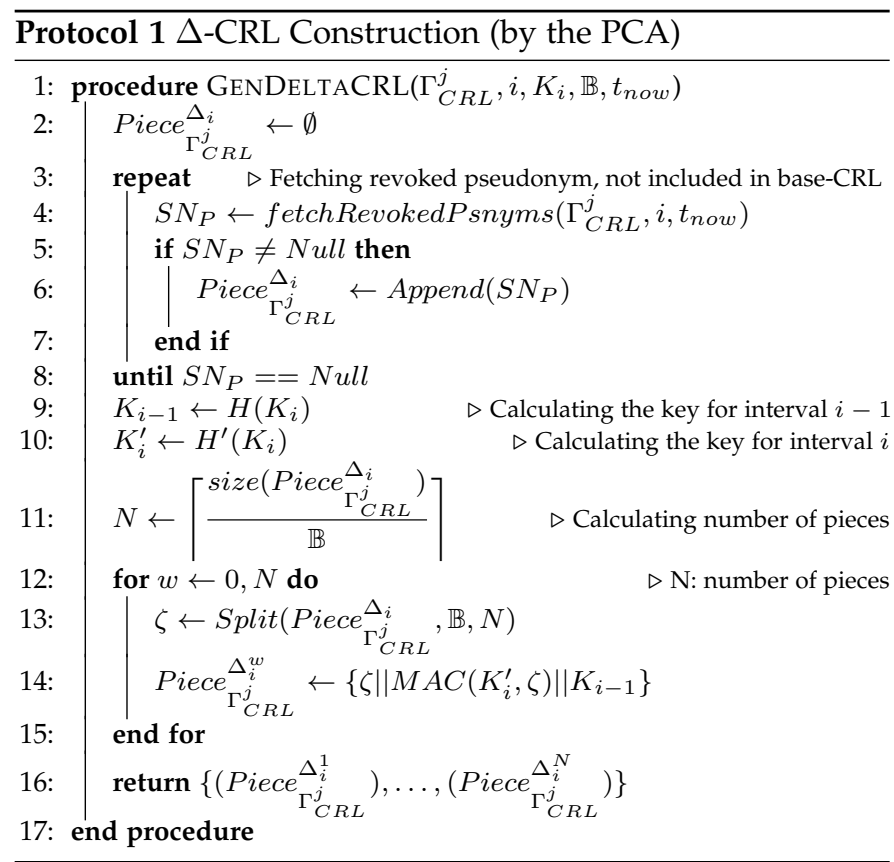

\subsection{2 $\Delta$-CRL Construction (Protocol 1 )}

Upon a new revocation event within a $\Gamma_{C R L}$ interval, the PCA distributes $\Delta$-CRL pieces to all vehicles. The number of revocation entries in a $\Delta$-CRL is proportional to the number of compromised vehicles and the number of revocation events. The PCA constructs $\Delta$-CRL pieces by including all recently revoked pseudonyms whose lifetimes are valid for the next pseudonym interval $\left(\tau_{P}^{i}\right)$ (steps $\left.1,2-18\right)$. The PCA then derives the corresponding MAC keys (steps $19-1$ 10) and it splits the $\Delta$-CRL into multiple pieces according to the maximum allocated bandwidth, i.e., system parameter $\mathbb{B}$, for CRL distribution (steps 111- 113). It then appends an authenticator to each $\Delta$-CRL piece by calculating $M A C\left(K_{i}^{\prime}\right.$, Piece $\left._{\Gamma_{C R L}^{j}}^{\Delta_{i}^{w}}\right)$ (step 114). Note that $\Delta$-CRLs for an interval $i$, i.e., $\tau_{P}^{i}$, are distributed within interval $i-1$, i.e., $\tau_{P}^{i-1}$, and the secret key $K_{i}^{\prime}$ is distributed upon pseudonym transition (from $\tau_{P}^{i-1}$ to $\tau_{P}^{i}$ ).

\subsubsection{CRL Subscription}

Each vehicle can receive necessary CRL pieces corresponding to its actual trip duration from nearby RSUs or neighboring vehicles. A vehicle broadcasts a signed query to its neighbors, to receive the missing pieces of the revocation information of $\Gamma_{C R L}^{i}$ during which the vehicle wishes to travel. Having received a CRL piece, it simply validates the piece by testing against the signed fingerprint (already obtained from RSUs or integrated in a subset of recently issued pseudonyms broadcasted in the network). If the BF test is successful, it accepts that piece and keeps requesting until successfully receiving all remaining pieces. A detailed protocol description is available in [41]. In case of $\Delta$-CRL, each vehicle should buffer all received $\Delta$-CRL pieces with appended MACs in order to validate them upon disclosure of the corresponding key. When the PCA discloses the key at every $\tau_{P}$, each vehicle computes the MAC of each piece

4. TESLA uses different hash functions to derive key $K_{i-1}$ and to compute MACs to mitigate potential vulnerabilities of using the same key for different cryptographic operations 60$]$.

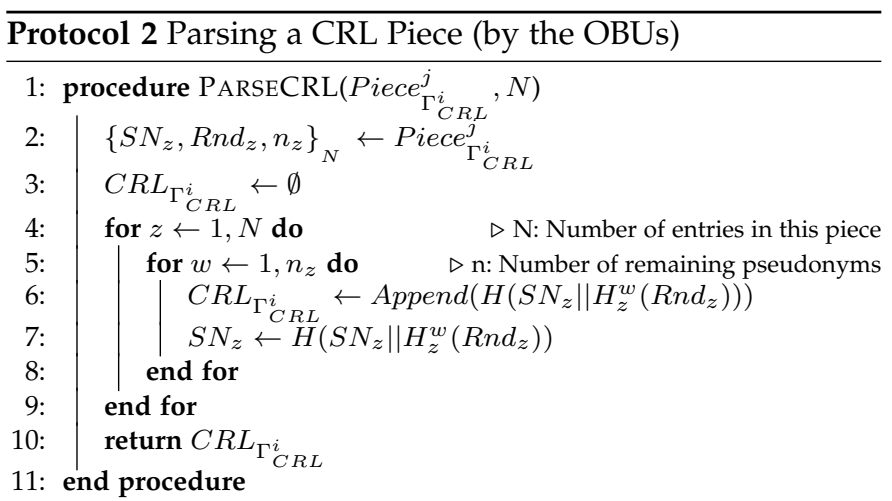

using that key. If the two MACs are the same, then the $\Delta$-CRL piece would be accepted; otherwise, dropped.

\subsubsection{Parsing CRL (Protocol[2)}

Upon reception and validation of a CRL piece, each vehicle derives the revoked pseudonym serial numbers from the obtained hash anchors, by calculating a hash value $n$ times: $H\left(S N_{z} \| H_{z}^{w}\left(\operatorname{Rnd}_{z}\right)\right.$ (steps 22-210). Revocation entries can be stored in local storage, and searched with $O(\log (n))$ time complexity. To enhance revocation status validation, a vehicle could generate a BF locally $[18 \mid$ with constant computational cost $(O(1))$ for insertions and search operations but at a cost of a false positive rate. Note that the search operation is very efficient because revocation entries are sorted for the period they are valid for, i.e., in a $\tau_{P}$ interval.

\section{Scheme Analysis and Evaluation}

For an analysis on how our scheme satisfies the security and privacy requirements, as well as operational requirements, we refer interested readers to [41]. In this section, we expand the security and privacy analysis for the $\Delta$-CRL distribution and the CRL fingerprint mechanisms. Then, we quantitatively demonstrate the efficiency, scalability, and resiliency of our vehicle-centric scheme, extending the results with respect to [41], through an extensive experimental evaluation.

\subsection{Qualitative Analysis}

$\triangle$-CRL distribution: Upon a new revocation event and $\Delta$-CRL release, an external adversary can try to link the recently revoked pseudonyms backwards, i.e., towards the beginning of the $\Gamma$ interval. However, due to the utilization of a hash chain during the pseudonym issuance process [41], it is infeasible to link a revoked pseudonym to the previously non-revoked pseudonyms. Moreover, vehicles can be loosely synchronized with the VPKI clock, e.g., through Global Positioning System (GPS) used to synchronize OBU clocks, in order to be informed about the time of key disclosure (by the PCA). Furthermore, each vehicle does not disclose the trip duration. Rather, each vehicle only requests for obtaining CRL pieces for the current $\Gamma_{C R L}$, which is a proto$\mathrm{col} / \mathrm{scheme}$ selectable parameter. More so, the requests are anonymized, i.e., signed by the currently valid pseudonym. For the next $\Gamma_{C R L}$, each vehicle changes its pseudonym and requests to obtain CRL pieces for the new interval using a new pseudonymous identifier. The pseudonyms are fully unlinkable, and there is no memory or linkage across such 
$\Gamma_{C R L}$ intervals. The signed requests do not reveal the actual identity of their owner, thus it does not harm user privacy.

Upon releasing $\Delta$-CRLs, vehicle buffer all received pieces with the appended MACs in order to validate them when the secret key is disclosed. However, an attacker could aggressively broadcast fake pieces of $\Delta$-CRL to mount a clogging DoS attack. The longer the pseudonym lifetimes are, the higher the frequency of broadcast is, and the higher the number of adversaries is, the larger the storage space needed. For example, if there is an adversary broadcasting bogus pieces of $\Delta$-CRL (with $\mathbb{B}=50 \mathrm{~KB} / \mathrm{s}$ and $\tau_{P}=5$ $\mathrm{min}$ ), then each neighboring vehicle needs $\approx 15 \mathrm{MB}$ of memory to store bogus CRL pieces. One can apply a rate limiting mechanism by requesting the "suspicious" node to piggyback a fingerprint of the $\Delta$-CRL pieces in its successive CAMs; otherwise, all received packets from the suspicious sender would be dropped. As CAMs are already signed, any suspicious behavior could be reported to the VPKI for further investigation.

Reversible pseudonym revocation status: When a misbehavior detection authority identifies a misbehaving vehicle, it queries the RA to initiate a resolution and revocation process. The RA queries the corresponding PCA to retrieve the ticket, used to obtain that pseudonym. Assume a vehicle has obtained pseudonyms for a period, e.g., a week or a month. The RA then progressively interacts with the corresponding H-LTCA towards obtaining all subsequent tickets issued for that vehicle ${ }^{5}$. The RA queries the PCAs towards revoking the pseudonyms issued for a given ticket ${ }^{6}$ In case of resolving the stated misbehavior, the RA informs the H-LTCA to issue more tickets for that vehicle. Moreover, the RA stops delivering a ticket (acquired from the H-LTCA during pseudonym resolution process [43]) to the PCAs towards revoking the pseudonyms issued for that ticket. In this way, a misbehaving entity can be temporarily evicted form the VC system; furthermore, upon resolving the issue, the entity can re-enter the system by leveraging the previously obtained pseudonyms without necessarily obtaining a fresh batch of pseudonyms. This flexibility also allows a more efficient and effective CRL distribution; at the same time, this prevents from overloading the VPKI entities from issuing unnecessary new sets of pseudonyms.

Our vehicle-centric scheme protects user privacy due to separation of duties: no single VPKI entity could fully de-anonymize a user by identifying the actual identity of or link successive pseudonyms belonging to a (partially) evicted vehicle. Collusion by an RA and a PCA does not reveal any information to link the users (long-term identities) with their pseudonyms because the tickets, issued by a H-LTCA, are anonymized and they do not reveal their owners' identities. Collusion by an RA and the H-LTCA does not enable them towards linking the corresponding pseudonyms: time-aligned pseudonyms are issued for all vehicles, thus there is no distinction among pseudonym sets. Upon a misbehavior event, the H-LTCA could only infer

5. In case of a multi-domain VC environment, one more step is required, i.e., the RA interacts with all Foreign-LTCAs (F-LTCAs) [43].

6. Using a ticket, one cannot identify the targeted PCA |43|; thus, the RA queries all PCAs in a domain to revoke pseudonyms issued for that ticket. This strongly protects user privacy: collusion by an RA and all PCAs does not reveal any information to harm user privacy [43].

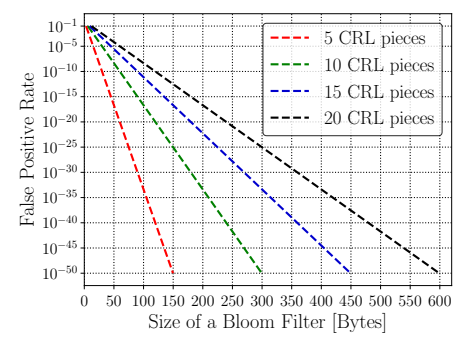

(a) Vehicle-centric scheme (b) Precode-and-hash scheme 34

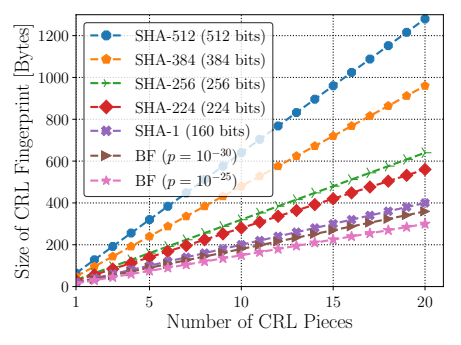

Fig. 4. Extra overhead for $C R L$ fingerprints.

that a user was evicted for an interval without any other information towards linking, thus tracking, a vehicle. For a detailed information held by each honest-but-curious VPKI entity, we refer readers to [43].

Synchronization with the VPKI clock: Lack of synchronization between the vehicles and the VPKI clock could affect integrity of the $\Delta$-CRL distribution. More precisely, in case of drifting clocks of the some victim vehicles, an adversary can manipulate a CRL piece. Upon disclosing the cryptographic key to compute the MAC, the adversary crafts a bogus CRL piece, e.g., excluding revoked pseudonym serial numbers from a CRL piece or adding valid pseudonyms into a fake CRL piece, and broadcast to the victim vehicles whose clock mildly drift from the VPKI clock. Due to lack of synchronization, such vehicles would accept the bogus pieces. In order to mitigate such a misbehavior, it suffices to have vehicles periodically synchronizing their clocks with the VPKI clock. For example, if the accuracy of an Real Time Clock (RTC) is 20 parts-per-million (ppm), i.e., $20 \times 10^{-6}$, and the maximum accepted error in timestamp is $1 \mathrm{sec}$, then each vehicle should synchronize its clock every 13.8 hours $\left(\frac{1 \times 10^{3} \mathrm{sec}}{20 \times 10^{-6} \mathrm{ppm}}\right)$, i.e., twice a day, which seems to be practical.

Vulnerability window: Even though our vehicle-centric scheme distributes CRLs and $\Delta$-CRLs, there could be a small vulnerability window. For example, when a new vehicle joins the system within the current $\tau_{p}$ interval, the PCA has already disclosed the cryptographic key corresponding to that interval; thus, that vehicle cannot rely on the received $\Delta$-CRL pieces because the key is already disclosed and the $\Delta$-CRL pieces could have been manipulated. Obviously, the shorter the pseudonym lifetimes are, the narrower the vulnerability window is. In general, there is a trade-off between closing down the vulnerability window and cost, notably communication overhead and deploying uninterrupted connectivity to the VPKI, e.g., dense deployment of RSUs or leveraging cellular communications. Depending on the type of misbehavior and the fraction of recently joined vehicles, the VPKI could opt in to geo-cast the signed revocation information at any point in time, even within a pseudonym lifetime. Alternatively, vehicles could request the neighboring RSUs or vehicles for the signed $\Delta$-CRL pieces. Note that fully closing down the vulnerability window requires an efficient revocation scheme combined with a persistent and reliable connectivity to the VPKI entities, e.g., leveraging cellular-based V2X communications [62].

CRL and fingerprint size comparison: Representing CRL pieces in a space-efficient BF trades off communication overhead for a false positive rate $(p)$. Fig. 4 a shows that the 
BF size linearly increases as the false positive rate decreases. For example, for $10 \mathrm{CRL}$ pieces covering one $\Gamma_{C R L}$ interval, and $p=10^{-20}$ (with the optimal number of hash functions), the BF size and thus the overhead for each pseudonym is 120 bytes. This eliminates the need to sign each CRL piece.

The PCA can concatenate the hash values for each CRL piece [34]. Fig. 4 $\mathrm{b}$ compares our BF-based CRL fingerprint size with the five approved hash algorithms [63]: SHA-1, SHA-224, SHA-256, SHA-384 and SHA-512, each producing hash digest size of 160, 224, 256, 384 and 512 bits, respectively. For instance, by employing precode-and-hash [34] with SHA1 (20 bytes output size) [34], the size of a fingerprint for 20 CRL pieces becomes 400 bytes; whereas employing our scheme results in an extra overhead of 311 bytes $\left(p=10^{-25}\right)$ or 371 bytes for the extremely low false positive rate $\left(p=10^{-30}\right)$. Alternatively, one can utilize truncated hash digests; however, truncated message digest must be carefully used: if the message digest length is too small, computation of pre-image, second pre-image or collisions becomes feasible [64]. All in all, truncation will not guarantee the expected security strength of a hash digest [63]. A detailed CRL size comparison with Compressed CRL (C $\left.{ }^{2} R L\right)$ scheme [12], [25], [26] can be found in our earlier work [41].

Compromising the security of the CRL fingerprint: A $\mathrm{BF}$ is a space-efficient probabilistic data structure that is used for efficient membership query. It essentially provides condensed authenticators for the inserted items at the cost of false positives. Fig. 5 illustrates a BF with insertion and query operations. In order to insert an item, e.g., $x$, into the $\mathrm{BF}$, we feed item $x$ into $k$ different hash functions $\left\{h_{1}, \ldots, h_{k}\right\}$ to identify the corresponding bits of the BF, i.e., $I_{x}=\left\{h_{1}(x) \bmod m, \ldots, h_{k}(x) \bmod m\right\}$. In this example, the size of the $\mathrm{BF}, m$, is 22 bits and the number of hash functions, $k$, is 3 . Items $\mathrm{y}$, and $\mathrm{z}$ are also inserted into the $\mathrm{BF}$. In order to query an item to check if it belongs to the $\mathrm{BF}$, we check if the item has been inserted into the BF by feeding it to the same hash functions. In this example, $x^{\prime}$ does not belong to the BF; $y^{\prime}$ is equal to $y$ and thus it exists. However, $z^{\prime}$ appears to be in the set while it has never been added, i.e., a false positive. The false positives arise due to the collision on the condensed hash digests and one can adjust the size of the $\mathrm{BF}$ to achieve a desired false positive rate [36], [65].

There are three types of attacks applicable to $\overline{\mathrm{BF}}$ [64] chosen-insertion attack, query-only attack, and deletion attack. In the chosen-insertion attack, an adversary can either add a new item into the $\mathrm{BF}$, or he could make the $\mathrm{BF}$ constructor, i.e., the PCA, insert a new item. In the query-only attack, an adversary targets the false positive rate of a BF towards generating a fake CRL piece to be accepted as legitimate. Finally, in the deletion attack, an adversary tries to delete an item, or make the PCA delete it from the BF. Note that this type of attack is for a specific form of BFs which allows deleting an item, e.g., counting BFs [65].

The chosen-insertion and query-only attacks are not applicable in our vehicle-centric CRL distribution scheme exactly because the BF is explicitly signed by the PCA, or it is integrated into a set of recently issued pseudonyms, i.e., implicitly signed. However, the query-only attack is applicable: an adversary could try to generate a bogus CRL piece in order to exclude revoked pseudonym serial numbers or add valid ones by forging a fake CRL piece that passes

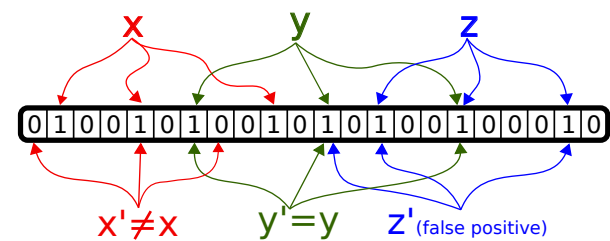

Fig. 5. BF insertion and query ( $m=22$ bits, $k=3$ hash functions, $n=3$ items).

the BF test. Note that this is different from a pollution or a DDoS attack: not only would it prevent a legitimate vehicle from obtaining a genuine CRL piece, but also disseminate an authentic-looking piece that passes the BF test. In fact, such an attack relies on sheer computational power; its effectiveness depends on the computational resources allocated. The probability of generating a bogus CRL piece, i.e., obtaining a false positive, is [35], |36]:

$$
P=\left[1-\left(1-\frac{1}{m}\right)^{k n}\right]^{k}
$$

Our scheme resists such attacks that attempt to exclude revoked pseudonym serial numbers or add valid ones by forging a fake CRL piece that passes the BF test $]^{7}$ An adversary could buy top-notch bitcoin-mining hardware, Antminer-S9 [66] $(14 \mathrm{TH} / \mathrm{s}, \$ 3,000)$. If $\Gamma_{C R L}=1$ hour and $p=10^{-20}$, and the optimal number of hash functions, $K=67$, the adversary needs 132,936 Antminer-S9 (\$400M) to generate a bogus piece within a $\Gamma_{C R L}$ interval $\left(\frac{10^{20} \times 67}{14 \times 10^{12}}\right)$. Alternatively, he could join AntPool [67], one of the largest Bitcoin mining pools, $(1,604,608 \mathrm{TH} / \mathrm{s})$ to generate a fake piece. Fig. 6 shows the time to conduct the query-only attack towards generating a bogus CRL piece. If $\Gamma_{C R L}=1$ hour and $p=10^{-20}$, and the optimal number of hash functions, $K=67$, the adversary could generate a bogus piece within 70 min, which might seem a practical threat. However, if $p=10^{-22}$ (with $K=73$ ) or even $p=10^{-23}$ (with $K=76)$, the adversary would need 5 or 55 days, respectively $\left(\frac{10^{22} \times 73}{1.6 \times 10^{18}}=126 h, \frac{10^{23} \times 76}{1.6 \times 10^{18}}=1,319 h\right)$. With inherently short $\tau_{P}$ (important for unlinkability and thus privacy) and $\Gamma_{C R L}$ interval, proper choice of $p$ makes attacks infeasible; in other words, irrelevant, as forged pieces refer to already expired credentials. Upon receiving conflicting pieces, vehicles report misbehavior to the VPKI to take appropriate actions, e.g., adjusting $p$. The results of our experiments in Sec. 5.2 rely on $p=10^{-30}$ and $K=100$.

Under certain circumstances, the chosen-insertion attack can also be applicable in our vehicle-centric CRL distribution scheme. For example, integrating CRL fingerprints into pseudonyms would result in larger pseudonym size, i.e., increasing packet size and overhead, thus higher channel congestion and error rates [4], [68]. In such cases, the size of a CRL fingerprint should not exceed a certain amount of bytes for efficiency reasons. Thus, the PCA cannot generate a CRL fingerprint with the desired size and false positive rate to be integrated into a subset of pseudonyms. This

7. Generating a fake BF (e.g., $p=10^{-20}$ ) with completely different valid pseudonyms serial number necessitates accessing at least, e.g., $10^{20}$, valid pseudonyms, i.e., a more powerful adversary (malicious VPKI entities), and is beyond the scope of our adversarial model. 


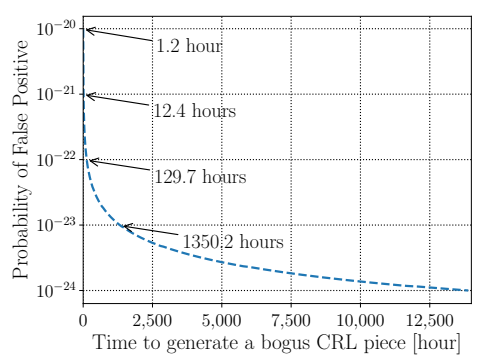

Fig. 6. Query-only attack on the CRL fingerprints; adversary's computational power is $1.6 \times 10^{18} \mathrm{TH} / \mathrm{sec}$.

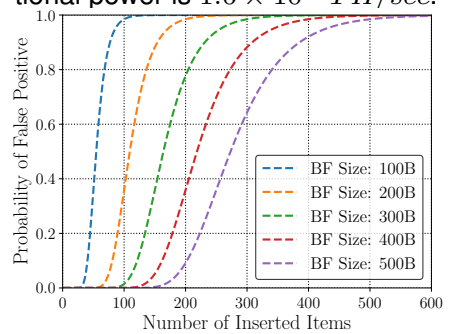

(a)

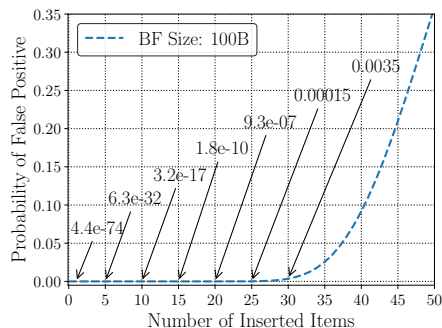

(b)
Fig. 7. Chosen-insertion attack on the CRL fingerprint.

implies that the PCA should insert more items (CRL pieces) into the CRL fingerprint with 'constant' size, and thus increase the probability of false positive. Fig. 7 a shows that the probability of generating a bogus CRL piece grows exponentially when the BF size is constant. Fig. $7 \mathrm{~b}$ shows that the probability of false positive when BF size is 100 bytes. The probability of false positive by inserting 5 items in the $\mathrm{BF}$ is $6.3 \times 10^{-32}$. Even with an extremely computationally powerful adversary, generating a bogus CRL piece is practically infeasible. However, by inserting 10 CRL pieces into the $\mathrm{BF}$ (assuming the BF size remains constant), the probability of false positive becomes $3.2 \times 10^{-17}$. By considering the computational power of an adversary to be $1.6 \times 10^{18}$ the time to generate a bogus CRL piece (with $K=67$ ) becomes $\approx 14$ seconds.

Depending on the type of misbehavior and revocation rate $(\mathbb{R})$, the number of revocation entries within a $\Gamma_{C R L}$ interval could be huge, which yields a larger CRL size. If this would result in a high rate of false positive, the PCA could opt in enlarging the maximum bandwidth $(\mathbb{B})$ for CRL distribution, or decreasing the $\Gamma_{C R L}$ interval (if $\Gamma_{C R L}$ is larger than $\Gamma$ ). Alternatively, the PCA could decrease the percentage of fingerprint-carrier pseudonyms; even with $1 \%$ of the vehicles to be fingerprint-carrier nodes, all of the vehicles could obtain the CRL fingerprint in time [41] In our experiments, even with $\mathbb{R}=5 \%, \mathbb{B}=50 \mathrm{~KB} / \mathrm{s}$, and probability of false positive $p=10^{-30}$, the number of CRL pieces becomes 7, with a 126-byte fingerprint.

\subsection{Quantitative Analysis}

\subsubsection{Experimental Setup}

We use OMNET++ [69] and the Veins framework to simulate a large-scale scenario using SUMO [70] with a realistic mobility trace, the LuST dataset |71|. For the cryptographic protocols and primitives (Elliptic Curve Digital Signature Algorithm (ECDSA)-256 and SHA-256 as per IEEE 1609.2 [4] and ETSI [1]), we use OpenSSL. V2X communication is IEEE
TABLE 2

Simulation parameters (LuST dataset).

\begin{tabular}{|c|c||c|c|}
\hline Parameters & Value & Parameters & Value \\
\hline \hline CRL/Fingerprint TX interval & $0.5 \mathrm{~s} / 5 \mathrm{~s}$ & Pseudonym lifetime & $30 \mathrm{~s}-600 \mathrm{~s}$ \\
\hline Carrier frequency & $5.89 \mathrm{GHz}$ & Area size & $15 \mathrm{KM} \times 15 \mathrm{KM}$ \\
\hline TX power & $20 \mathrm{~mW}$ & Number of vehicles & 138,259 \\
\hline Physical layer bit-rate & $18 \mathrm{Mbps}$ & Number of trips & 287,939 \\
\hline Sensitivity & $-89 \mathrm{dBm}$ & Average trip duration & $692.81 \mathrm{~s}$ \\
\hline Thermal noise & $-110 \mathrm{dBm}$ & Duration of simulation & 4 hour $(7-9,17-19)$ \\
\hline CRL dist. Bandwidth $(\mathbb{B})$ & $5-100 \mathrm{~KB} / \mathrm{s}$ & $\Gamma$ & $1-60 \mathrm{~min}$ \\
\hline Number of RSUs & 100 & $\Gamma_{C R L}$ & $60 \mathrm{~min}$ \\
\hline
\end{tabular}

TABLE 3

Vehicle-centric revocation information for LuST dataset $(\mathbb{R}=1 \%, \mathbb{B}=10 \mathrm{~KB} / \mathrm{s})$

\begin{tabular}{|c||cccc|}
\hline $\begin{array}{c}\text { Pseudonym } \\
\text { Lifetime }\end{array}$ & $\begin{array}{c}\text { Number of } \\
\text { Psnyms }\end{array}$ & $\begin{array}{c}\text { Number of } \\
\text { Revoked Psnyms }\end{array}$ & $\begin{array}{c}\text { Average } \\
\text { Number per } \Gamma_{C R L}\end{array}$ & $\begin{array}{c}\text { Number of } \\
\text { Pieces }\end{array}$ \\
\hline \hline$\tau_{P}=30 \mathrm{~s}$ & $3,425,565$ & 34,256 & 1,428 & 13 \\
\hline$\tau_{P}=60 \mathrm{~s}$ & $1,712,782$ & 17,128 & 714 & 7 \\
\hline$\tau_{P}=300 \mathrm{~s}$ & 342,556 & 3,426 & 143 & 2 \\
\hline$\tau_{P}=600 \mathrm{~s}$ & 171,278 & 1,713 & 72 & 1 \\
\hline
\end{tabular}

TABLE 4

Simulation parameters for LuST dataset $\left(\tau_{P}=60 s\right)$.

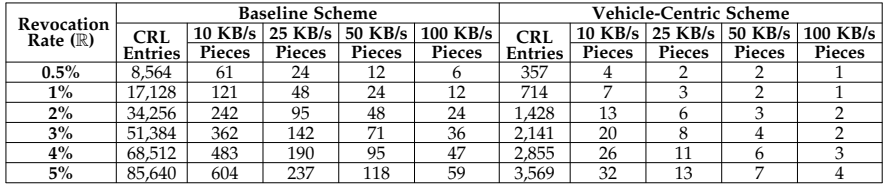

802.11p [72] and cryptographic protocols and primitives were executed on a virtual machine (dual-core 2.0 GHz). To evaluate CRL pieces construction (with a BF) and validate CRL pieces (BF membership check), we used a Nexcom vehicular OBU (Dual-core 1.66 GHz, 1GB memory) from the PRESERVE project [9]. For CRL fingerprint operations, BF insertion and query, we used PYBLOOM [73].

Effective placement of the RSUs: We sorted the intersections with the highest numbers of vehicles passing by [74]. We then placed the RSUs based on these "highly-visited" intersections with non-overlapping radio ranges.

Metrics: We evaluate the latency to obtain the latest CRL pieces, i.e., from the time a vehicle enters the system until it successfully downloads them. We choose a small amount of bandwidth $(\mathbb{B})$ for the distribution, e.g., $5-10 \mathrm{~KB} / \mathrm{s}$. Note that request-triggered CRL piece broadcasts at 5-100 KB/s (40-800 $\mathrm{Kbit} / \mathrm{s}$ ) are practical because $802.11 \mathrm{p}$ supports data-rates up to $24 \mathrm{Mbit} / \mathrm{s}$ |72].

Table 2 shows the simulation parameters; Tables 3 and 4 show the simulation information for the LuST dataset with respect to different pseudonyms lifetimes $\left(\tau_{P}\right)$, revocation rates $(\mathbb{R})$, and maximum bandwidth for distributing CRL pieces $(\mathbb{B})$. We assume that the revocation events are uniformly distributed over a day. For example, if $\tau_{P}=60 \mathrm{~s}$, the total number of pseudonyms for one day is around $1.7 \mathrm{M}$. Assuming $1 \%$ of the pseudonyms are revoked $(\mathbb{R}=1 \%)$, there will be around $17 \mathrm{~K}$ revoked pseudonyms in a day. With our vehicle-centric approach, each vehicle only needs to obtain pieces of CRL for the interval it travels. When $\Gamma_{C R L}=1$ hour, the average number of entries per $\Gamma_{C R L}$ interval is around 710 . With $\mathbb{B}=10 \mathrm{~KB} / \mathrm{s}$, total number of pieces will be 6 . These numbers come from the actual implementation of encoded packets, serialized with the $\mathrm{C}++$ boost library. 


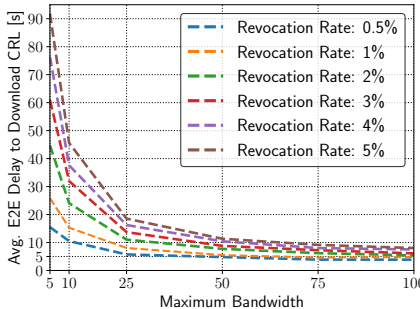

(a) Vehicle-centric scheme

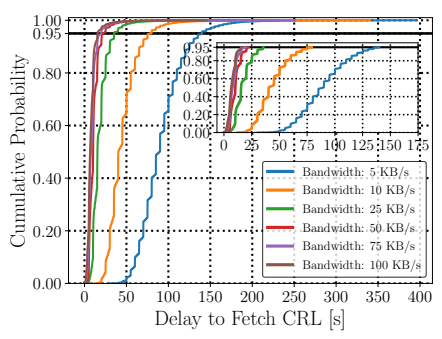

(b) Vehicle-centric scheme
Fig. 8. (a) Bandwidth-delay trade off $\left(\tau_{P}=60 \mathrm{~s}\right)$. (b) CDF of end-to-end delay with different bandwidth $\left(\tau_{P}=30 s, \mathbb{R}=5 \%\right)$.

\subsubsection{Summary of Results}

The results here complement (and are comparable with) the presented results in $[41]$ and they share the same configuration and system set up. Our vehicle-centric scheme converges more than 40 times faster than the state-of-theart [15], [16], [18], termed here the baseline scheme, with a similar experimental set up (Fig. $10 \mathrm{~b}$ ). Moreover, with the baseline scheme, the number of vehicles that successfully obtained the latest CRL, referred to as cognizant vehicles, is highly dependent on the revocation rate and it significantly drops when the revocation rate increases from $0.5 \%$ to $5 \%$. However, the performance of our scheme is not affected by the revocation rate: the number of cognizant nodes remains almost intact even if the revocation rate increases up to $5 \%$ [41]. Furthermore, our vehicle-centric scheme is more resilient to selfish ${ }^{8}$, pollution, and DoS attacks: with $25 \%$ of vehicles in the baseline system compromised, one could prevent almost all legitimate vehicles from obtaining the CRLs; however, with our scheme, the percentage of informed vehicles remains almost intact even if $50 \%$ of the vehicles are compromised (Fig. 11, 12, 13, 14.

Furthermore, our experiments show that the distribution of $\Delta$-CRL pieces as well as the validation keys are efficient and resilient against DoS attacks; more specifically, $95 \%$ of the vehicles received the $\Delta$-CRL pieces in less than $52 \mathrm{~s}$ : $F_{x}(t=52 \mathrm{~ms})=0.95$, and $95 \%$ of them obtained the validation keys within less than 31s: $F_{x}(t=31 \mathrm{~ms})=0.95$ (Fig. 9 . Moreover, BF construction and membership checks are much more efficient than the baseline scheme: the average latency to verify a CRL piece for the baseline scheme (i.e., verifying an ECDSA-256 bits signature) is $2.346 \mathrm{~ms}$. However, for the vehicle-centric scheme, the latency to validate a CRL piece using a $\mathrm{BF}$ (with probability of false positive rate $p=10^{-25}$ and $K=67$, as the optimal number of hash functions) is $0.352 \mathrm{~ms}$, i.e., 6.6 times faster than the baseline scheme (Table 5 and Table 6. Finally, our experiments confirm that our scheme outperforms the baseline scheme in terms of communication overhead (notably security overhead) [41].

\subsubsection{Vehicle-Centric CRL Distribution Evaluation}

Fig. 8 a shows the average end-to-end latency to obtain the CRLs as a function of maximum bandwidth for the vehiclecentric scheme. The delays were averaged over vehicles operating during rush hours. The total number of pseudonyms is

8. Such nodes do not perform any "active" attack, e.g., a clogging DoS attack, rather they become silent and they never respond to a $\mathrm{CRL} / \Delta$-CRL piece request.

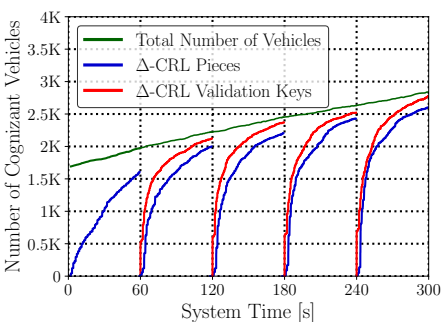

(a) 7:05-7:10 am $(\mathbb{B}=10 \mathrm{~KB} / \mathrm{s})$

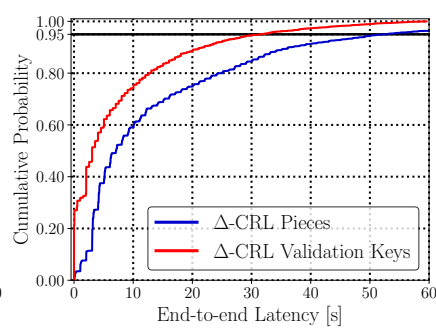

(b) 7:05-7:10 am $(\mathbb{B}=10 \mathrm{~KB} / \mathrm{s})$
Fig. 9. End-to-end delay to fetch $\Delta$-CRL pieces and validation keys for vehicle-centric scheme $\left(\tau_{P}=60\right.$ sec., $\left.\mathbb{R}=5 \%, \gamma_{\text {key }}=0.5, \gamma_{\text {piece }}=2\right)$.

$1.7 \mathrm{M}\left(\tau_{P}=60 \mathrm{~s}\right)$ and the maximum bandwidth ranges from 5 to $100 \mathrm{~KB} / \mathrm{s}$. In general, the smaller amount of bandwidth for CRL distribution and the higher the revocation rate are, the higher the latency for all vehicles to obtain the CRL. For example, the average latency, with $\mathbb{R}=5 \%$, decreases from $45.68 \mathrm{~s}$ to $18.48 \mathrm{~s}$ as the $\mathbb{B}$ increases from 10 to $25 \mathrm{~KB} / \mathrm{s}$. It is imperative to allocate as low bandwidth as possible without compromising the timely distribution of CRLs. On the one hand, allocating lower bandwidth for the CRL distribution diminishes interference with the safety-critical operations and mitigates pollution and DoS attacks; but, it degrades the timely CRLs distribution. On the other hand, allocating large bandwidth would enhance timely CRLs distribution at the cost of interference with safety operations and/or enabling an attacker to broadcast a fake CRL piece at a high rate. Depending on the revocation rates (i.e., events that lead to revocation), the optimal bandwidth for CRL distribution can be properly determined to achieve a certain level of quality of service without compromising the operation of time-critical messages. For example, with $\mathbb{R}=5 \%$, one can increase the bandwidth from $25 \mathrm{~KB} / \mathrm{s}$ to $100 \mathrm{~KB} / \mathrm{s}$ to reduce the delay from 18.48 s to 8 s, i.e., 2.3 faster CRL distribution.

Fig. 8 b shows the Cumulative Distribution Function (CDF) of end-to-end latencies to obtain the needed CRLs with different bandwidths for the vehicle-centric scheme. In general, the larger the allocated bandwidth for CRL distribution, the less the average latency to obtain CRLs. For example, with $\mathbb{R}=5 \%$ and $\mathbb{B}=50 \mathrm{~KB} / \mathrm{s}, 95 \%$ of the vehicles received the needed pieces of the CRL in less than 24s: $F_{x}(t=24 m s)=0.95$, i.e., $\operatorname{Pr}\{t \leq 24 m s\}=0.95$.

\subsubsection{Vehicle-Centric $\Delta$-CRL Distribution Evaluation}

Upon a new revocation event in a $\Gamma_{C R L}$ interval, the PCA constructs $\Delta$-CRL pieces by including the recently revoked pseudonyms (not included in the base-CRL). We emulate revocation events, e.g., due to malfunctioning of sensors, in every pseudonym lifetime with $\mathbb{R}=5 \%$, i.e., $5 \%$ of the pseudonyms within each $\tau_{P}$ should be revoked and included in the $\Delta$-CRL pieces. The total number of pseudonyms is $1.7 \mathrm{M}\left(\tau_{P}=60 \mathrm{~s}\right)$ and the maximum bandwidth to distribute $\Delta$-CRL pieces is up to $10 \mathrm{~KB} / \mathrm{s}$. In this experiment, $\Delta$-CRL pieces are broadcasted with frequency $\gamma_{\text {piece }}=2$ (one piece every $2 \mathrm{sec}$.) and validation keys are broadcasted with frequency $\gamma_{k e y}=0.5$ (2 times per second). We evaluate the latency to obtain $\Delta$-CRL pieces and validation keys. The longer the pseudonyms lifetimes combined with higher frequency of broadcasts (and larger coverage of the area by 


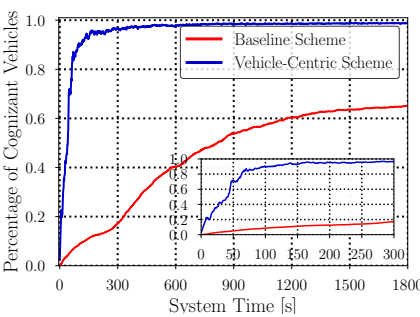

(a) 7:00-7:10 am $(\mathbb{B}=25 \mathrm{~KB} / \mathrm{s})$

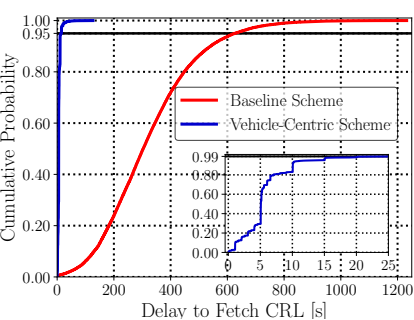

(b) 7-9 am, 5-7 pm $(\mathbb{B}=25 \mathrm{~KB} / \mathrm{s})$
Fig. 10. End-to-end delay to fetch CRLs $\left(\tau_{P}=60 s, \mathbb{R}=1 \%\right)$.

RSUs), the faster the convergence time is, thus the narrower the revocation vulnerability window becomes.

Fig. 9 shows the performance of the vehicle-centric scheme for the $\Delta$-CRLs distribution. A new revocation event happens at the beginning of a pseudonym lifetime and $5 \%$ of pseudonyms should be revoked. The PCA constructs $\triangle$-CRL pieces and the RSUs broadcast them. As illustrated in Fig. 9 a, the majority of vehicles could obtain $\Delta$-CRL pieces until the end of each pseudonym lifetime. Upon a pseudonym transition, the validation key for the $\Delta$-CRL pieces is released. However, there is a time lag until practically all vehicles are informed about the validation key disclosure. To ensure that every vehicle has already obtained the validation keys at the beginning of each $\tau_{P}$, the PCA could distribute the $\Delta$-CRL validation keys in the middle of preceding $\tau_{P}$. In other words, in order to optimize the distribution of $\Delta-C R L$ pieces to be used during $\tau_{P}^{i}$, the PCA would start distributing the $\Delta$-CRL pieces from the middle of $\tau_{P}^{i-2}$ until the middle of $\tau_{P}^{i-1}$; accordingly, the distribution of validation key, corresponding to $\tau_{P}^{i}$, would be started from the middle of $\tau_{P}^{i-1}$.

Fig. 9 b shows the CDF of delays for distributing $\Delta-C R L$ pieces and the validation keys. For the $\Delta-C R L$ pieces distribution, $F_{x}(t=52 \mathrm{~ms})=0.95$ while for the $\Delta$-CRL validation key distribution, $F_{x}(t=31 \mathrm{~ms})=0.95$. Faster convergence of $\Delta$-CRL validation keys, in comparison with $\Delta$-CRL pieces, stems from potentially multiplicity of $\Delta$-CRL pieces and the frequency of distribution $(\gamma)$. In order to mitigate a memoryexhaustion DoS attack on $\Delta$-CRL pieces distribution, we mandate a rate limiting mechanism. This ensures that a compromised insider cannot 'abuse' the allocated bandwidth towards performing a memory-exhaustion DoS attack on the distribution of $\Delta$-CRL pieces. Note that the distribution of $\Delta$-CRL validation keys is not vulnerable to a DoS attack |60|

\subsubsection{Performance Comparison}

We compare our scheme with the baseline scheme [15], [16] [18] that uses RSUs and car-to-car epidemic distribution, with the same assumptions, configuration, and system parameters. For the baseline scheme, the CA signs each CRL piece and can specify a "time interval" so that each vehicle receives $\mathbb{D}$ pseudonyms during the pseudonym acquisition process. As a result, for each batch of revoked pseudonyms, a single key $s_{i}$ (256 bit) is disclosed. Similarly, the PCA in our scheme can be configured to issue $\mathbb{D}$ pseudonyms per $\Gamma$, i.e., $\mathbb{D}=\frac{\Gamma}{\tau_{P}}$. To revoke a batch of $\mathbb{D}$ pseudonyms, the serial number of the first revoked pseudonym in the hash chain and a random number, each 256 bits long, are disclosed. For both schemes, we assume a fully-unlinkable pseudonym provisioning policy [43], i.e., $\Gamma=\tau_{P}=1 \mathrm{~min}$.

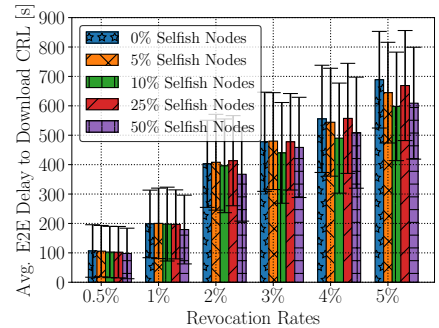

(a) Baseline scheme

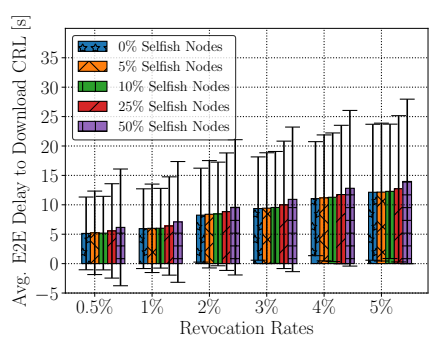

(b) Vehicle-centric scheme
Fig. 11. Resilience comparison against selfish nodes with different revocation rates $\left(7: 00-7: 30, \tau_{p}=30 s, \mathbb{B}=50 \mathrm{~KB} / \mathrm{s}\right)$.

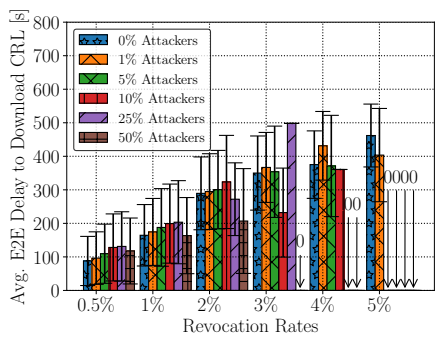

(a) Baseline scheme

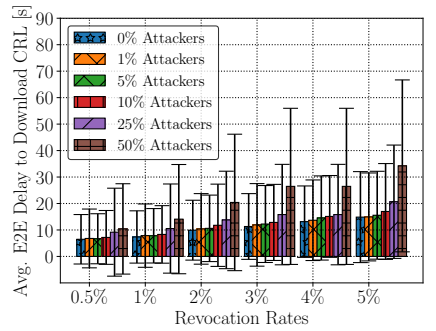

(b) Vehicle-centric scheme
Fig. 12. Resilience comparison against pollution and DoS attacks with different revocation rates $\left(7: 00-7: 10, \tau_{p}=30 s, \mathbb{B}=50 \mathrm{~KB} / \mathrm{s}\right)$.

We assume that vehicles are provided with enough pseudonyms corresponding to their actual trips for a day. Upon a revocation event, information on all revoked pseudonyms for the day is disseminated for the baseline scheme. In contrast, with our scheme, the CRL entries are distributed in a time prioritized manner, i.e., revoked pseudonyms whose validity intervals fall within the current $\Gamma_{C R L}$ interval. Moreover, by disseminating signed BF in advance, the verification cost is minimal compared to baseline signature verification, i.e., zero delay to verify the BF integrated in fingerprint-carrier pseudonyms or one signature verification for all CRL pieces. The processing delay to perform BF membership check is evaluated in Table 5 .

Fig. 10 a shows the number of cognizant vehicles over time for the two schemes. Vehicle-centric distribution of the CRL pieces converges faster: the number of cognizant vehicles is very close to the actual number of vehicles in the system. Fig. 10, b shows the CDF of delays for the two schemes: for the baseline, $F_{x}(t=626 s)=0.95$, whereas with our scheme, $F_{x}(t=15 s)=0.95$, i.e., converging more than 40 times faster. The principal reasons for such significant improvements are the prioritization of the revocation entries based on their validity intervals, thus a huge reduction in size, as well as the efficient verification of CRL pieces.

Fig. 11 shows the average end-to-end latency to successfully obtain the entire CRLs when a fraction of cognizant vehicles are considered to be selfish. Such nodes do not perform any "active" attack, e.g., a clogging DoS attack; rather, they become silent and they never respond to a CRL piece request. Fig. 11 shows the average end-to-end delay to obtain the entire CRL pieces in the presence of selfish nodes. For the baseline scheme, the average end-to-end latency linearly increases when the revocation rate increases; however, the average latency seems to be decreasing when the percentage of selfish nodes increases. The reason is that 


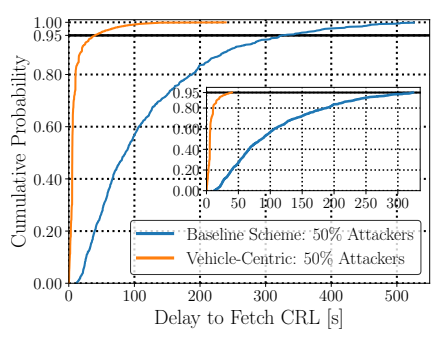

(a) CDF of delays under a DoS attack

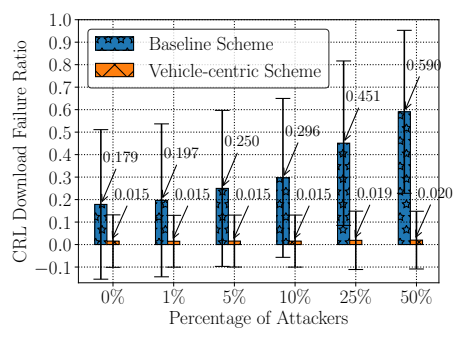

(b) Probability of failure
Fig. 13. (a) CDF of latency to successfully obtain CRL pieces $(50 \%$ attackers). (b) $C R L$ download failure ratio as a function of DoS attackers $\left(\tau_{P}=30 \mathrm{~s}, \mathbb{B}=50 \mathrm{~KB} / \mathrm{s}\right)$.

the probability of successfully obtaining the CRL decreases, i.e., the higher the revocation rate combined with the higher percentage of the selfish nodes, the less the average number of cognizant vehicles is (this becomes clear in Fig. 14). In contrast, when there are $50 \%$ selfish nodes and $\mathbb{R}=5 \%$, the end-to-end latency for our vehicle-centric scheme increases from 12.13 s to 14 s, i.e., $\approx 15 \%$ extra delay. Unlike performing a DoS attack that is detectable, i.e., identifying the source of a misbehaving node, detecting such a misbehavior is challenging; detecting and attributing misbehavior to trigger the revocation is beyond the scope of this work.

Fig. 12 shows the average end-to-end latency when attackers conduct pollution and DoS attacks by periodically broadcasting bogus CRL pieces once every $0.5 \mathrm{~s}$. The delays were averaged over vehicles successfully obtained CRL pieces. Fig. 12 a shows that the baseline scheme is adversely affected when the number of compromised vehicles increases. For example, when $\mathbb{R}=4 \%$ and $25 \%$ of the OBUs are compromised, no vehicle could successfully obtain the entire CRL pieces. In contrast, Fig. $12 \mathrm{~b}$ shows the performance of our vehicle-centric scheme: with $\mathbb{R}=4 \%$ and $25 \%$ of the OBUs misbehave in this way, the average end-to-end delay reasonably increases (from 13.13s to 15.81s).

Fig. 13 a shows the CDF of delays when $50 \%$ of the OBUs are compromised and periodically broadcast bogus CRL pieces once every $0.5 \mathrm{~s}$. For the baseline scheme, $F_{x}(t=$ $330 s)=0.95$, or $\operatorname{Pr}\{t \leq 330 s\}=0.95$, whereas with vehiclecentric scheme, $F_{x}(t=40 s)=0.95$, or $\operatorname{Pr}\{t \leq 40 s\}=0.95$. Fig. 13 b shows the probability of failure to obtain the CRL pieces for the baseline and vehicle-centric schemes against such a misbehavior. With the baseline scheme, the probability of failure to obtain CRL pieces increases from 0.18 to 0.59 when the percentage of DDoS attackers increases from zero to $50 \%$. This implies that the majority of the vehicles would reach their destination without successfully obtaining the CRL pieces. In contrast, for the vehicle-centric scheme, even if $50 \%$ of the OBUs are compromised and conduct a clogging DoS attack, the probability of failure is not considerably affected, in fact it is almost negligible, i.e., increasing from 0.015 to 0.020 . This shows the resiliency of our vehicle-centric scheme against DDoS attacks: the significant improvements that can be achieved by prioritization of the revocation entries based on their validity intervals, i.e., a huge reduction in size, thus significantly increased its resiliency against resource depletion attacks.

Fig. 14 shows the histogram of successfully received CRL pieces for the baseline scheme and the vehicle-centric scheme.

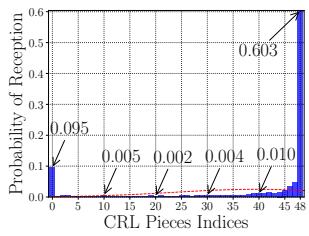

(a) Baseline: no attackers

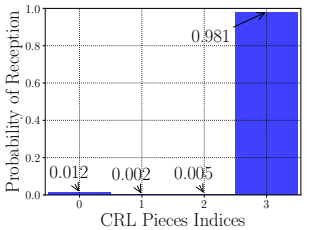

(d) Vehicle-centric: no attackers

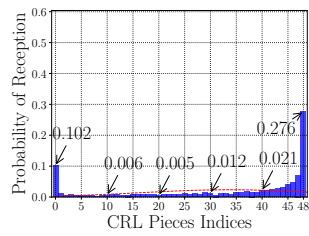

(b) Baseline: 10\% attackers

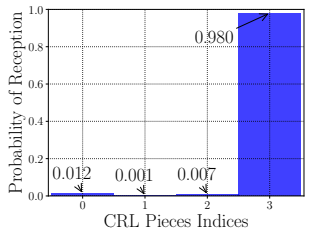

(e) Vehicle-centric: $10 \%$ attackers

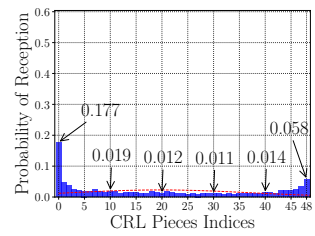

(c) Baseline: $50 \%$ attackers

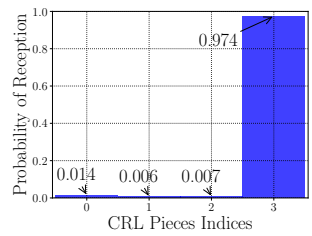

(f) Vehicle-centric: $50 \%$ attackers
Fig. 14. Probability of successful $C R L$ pieces reception $\left(\tau_{P}=30 \mathrm{~s}, \mathbb{B}=\right.$ $50 K \mathrm{~B} / \mathrm{s})$. (a) and (d): no attacks. (b), (c), (e), (f): under a DoS attack.

TABLE 5

Latency for validation all $C R L$ pieces using a $B F$, executed on a Nexcom OBU, averaged over $10 \mathrm{~K}$ runs $\left(\tau_{P}=60 \mathrm{~s}, \mathbb{B}=50 \mathrm{~KB} / \mathrm{s}\right)$.

\begin{tabular}{|c|c|c|c|c|}
\hline Revocation Rate & BF size & false positive & delay & check/sec. \\
\hline \hline \multirow{5}{*}{$1 \%$} & 252 bits & $\mathrm{p}=10^{-25}$ & $0.709 \mathrm{~ms}$ & 2,819 \\
& $261 \mathrm{bits}$ & $\mathrm{p}=10^{-26}$ & $0.729 \mathrm{~ms}$ & 2,741 \\
& $270 \mathrm{bits}$ & $\mathrm{p}=10^{-27}$ & $0.749 \mathrm{~ms}$ & 2,668 \\
& $282 \mathrm{bits}$ & $\mathrm{p}=10^{-28}$ & $0.776 \mathrm{~ms}$ & 2,577 \\
& $291 \mathrm{bits}$ & $\mathrm{p}=10^{-29}$ & $0.839 \mathrm{~ms}$ & 2,384 \\
& $300 \mathrm{bits}$ & $\mathrm{p}=10^{-30}$ & $0.859 \mathrm{~ms}$ & 2,329 \\
\hline \hline \multirow{5}{*}{$5 \%$} & $840 \mathrm{bits}$ & $\mathrm{p}=10^{-25}$ & $2.539 \mathrm{~ms}$ & 2,756 \\
& $957 \mathrm{bits}$ & $\mathrm{p}=10^{-26}$ & $2.613 \mathrm{~ms}$ & 2,678 \\
& $990 \mathrm{bits}$ & $\mathrm{p}=10^{-27}$ & $2.667 \mathrm{~ms}$ & 2,624 \\
& $940 \mathrm{bits}$ & $\mathrm{p}=10^{-28}$ & $2.774 \mathrm{~ms}$ & 2,522 \\
& 1067 bits & $\mathrm{p}=10^{-29}$ & $2.971 \mathrm{~ms}$ & 2,355 \\
& $1100 \mathrm{bits}$ & $\mathrm{p}=10^{-30}$ & $3.043 \mathrm{~ms}$ & 2,300 \\
\hline
\end{tabular}

With the baseline scheme, even if there is no attacker in the system, $60 \%$ of the vehicles could successfully obtain the CRL pieces within their trip duration. More interesting, when $50 \%$ of the OBUs are compromised and misbehaving by broadcasting periodically fake CRL pieces, only $6 \%$ of the vehicles could successfully obtain the entire CRL pieces. In contrast, with the vehicle-centric scheme, even if $50 \%$ of the vehicles misbehave, $97 \%$ of the vehicles would successfully obtain the entire CRL pieces within their trip duration. This shows that the operation of our vehicle-centric scheme is not considerably affected even if $50 \%$ of the OBUs are compromised, and the vehicles could still obtain the needed CRL pieces within a reasonable delay.

Table 5 shows the latency for validating all CRL pieces with different false positive rates. We performed our experiments on the Nexcom OBU boxes from the PRESERVE project $|9|$. For example, the latency to validate all CRL piece, with $\mathbb{R}=1 \%$ and $p=10^{-30}$, is $0.854 \mathrm{~ms}$. Table 6 shows the latency for inserting CRL pieces in a $\mathrm{BH}^{9}$ For example, the end-to-end delay to construct the CRL fingerprint, with $\mathbb{R}=1 \%$ and $p=10^{-30}$, is $1.14 \mathrm{~ms}$.

9. To directly compare the latencies, we conducted both experiments, i.e., BF insertion and membership check, on the Nexcom OBU boxes even though a PCA would have a stronger computational resource. 
TABLE 6

Latency for inserting $C R L$ pieces into a $B F$, executed on a Nexcom OBU, averaged over $10 \mathrm{~K}$ runs $\left(\tau_{P}=60 \mathrm{~s}, \mathbb{B}=50 \mathrm{~KB} / \mathrm{s}\right)$.

\begin{tabular}{|c|c|c|c|c|}
\hline Revocation Rate & BF size & false positive & delay & check/sec. \\
\hline \hline \multirow{5}{*}{$1 \%$} & 252 bits & $\mathrm{p}=10^{-25}$ & $0.949 \mathrm{~ms}$ & 2,108 \\
& $261 \mathrm{bits}$ & $\mathrm{p}=10^{-26}$ & $0.976 \mathrm{~ms}$ & 2,049 \\
& $270 \mathrm{bits}$ & $\mathrm{p}=10^{-27}$ & $1.004 \mathrm{~ms}$ & 1,991 \\
& $282 \mathrm{bits}$ & $\mathrm{p}=10^{-28}$ & $1.041 \mathrm{~ms}$ & 1,921 \\
& $291 \mathrm{bits}$ & $\mathrm{p}=10^{-29}$ & $1.113 \mathrm{~ms}$ & 1,796 \\
& $300 \mathrm{bits}$ & $\mathrm{p}=10^{-30}$ & $1.140 \mathrm{~ms}$ & 1,754 \\
\hline \hline \multirow{5}{*}{$5 \%$} & $840 \mathrm{bits}$ & $\mathrm{p}=10^{-25}$ & $3.334 \mathrm{~ms}$ & 2,099 \\
& 957 bits & $\mathrm{p}=10^{-26}$ & $3.432 \mathrm{~ms}$ & 2,039 \\
& $990 \mathrm{bits}$ & $\mathrm{p}=10^{-27}$ & $3.545 \mathrm{~ms}$ & 1,974 \\
& $940 \mathrm{bits}$ & $\mathrm{p}=10^{-28}$ & $3.662 \mathrm{~ms}$ & 1,911 \\
& $1067 \mathrm{bits}$ & $\mathrm{p}=10^{-29}$ & $3.898 \mathrm{~ms}$ & 1,796 \\
& $1100 \mathrm{bits}$ & $\mathrm{p}=10^{-30}$ & $3.977 \mathrm{~ms}$ & 1,760 \\
\hline
\end{tabular}

\section{Conclusion}

Paving the way for deploying secure and privacy-preserving VC systems, standardization bodies have reached an agreement towards deploying a special-purpose VPKI without reaching a consensus on how to efficiently and timely distribute CRLs in a large-scale environment. The success of secure and privacy-preserving VC systems requires effective mechanisms for distributing CRLs, to guarantee the operations of the systems. We proposed a practical framework to effectively distribute CRLs: our vehicle-centric scheme distributes necessary CRL pieces corresponding to a vehicle's targeted region and actual trip duration, i.e., obtaining only region- and time-relevant revocation information. Through extensive experimental evaluation, we demonstrated that our scheme is highly efficient and scalable, and it is resilient against selfish nodes, as well as pollution and DoS attacks. This supports that our scheme is a viable solution towards catalyzing the deployment of the secure and privacyprotecting VC systems.

\section{ACKNOWLEDGEMENT}

Work supported by the Swedish Foundation for Strategic Research (SSF) SURPRISE project and the KAW Academy Fellowship Trustworthy IoT project.

\section{RefERENCES}

[1] ETSI-TR-102-638, "Intelligent transport systems (its); vehicular communications; basic set of applications; definitions," ETSI, Tech. Rep., Jun. 2009.

[2] P. Papadimitratos, A. L. Fortelle, K. Evenssen, R. Brignolo, and S. Cosenza, "Vehicular Communication Systems: Enabling Technologies, Applications, and Future Outlook on Intelligent Transportation," IEEE Communications Magazine, vol. 47, no. 11, pp. 84--95, Nov. 2009.

[3] H. Jin, M. Khodaei, and P. Papadimitratos, "Security and Privacy in Vehicular Social Networks," in Vehicular Social Networks. Taylor \& Francis Group, 2016.

[4] IEEE-1609.2, "IEEE Standard for Wireless Access in Vehicular Environments - Security Services for Applications and Management Messages," Mar. 2016.

[5] Security-WG5, "Security \& Certification: Trust Models for Cooperative Intelligent Transport System (C-ITS), An analysis of the possible options for the design of the C-ITS trust model based on Public Key Infrastructure in Europe," https://smartmobilitycommunity. eu/sites/default/files/Security_WG5An1_v1.1.pdt. C-ITS Platform WG5, Jan. 2016.
[6] ------, "Security \& Certification: Revocation of Trust in Cooperative-Intelligent Transport Systems(C-ITS)," https://smartmobilitycommunity.eu/sites/default/files/ Security_WG5An2_v1.0.pdt C-ITS Plattorm WG5.

[7] PKI-Memo, "C2C-CC,"'http://www.car-2-car.org/. Feb. 2011.

[8] P. Papadimitratos, L. Buttyan, J.-P. Hubaux, F. Kargl, A. Kung, and M. Raya, "Architecture for Secure and Private Vehicular Communications," in IEEE International Conference on ITS Telecommunications (ITST), Sophia Antipolis, Jun. 2007, pp. 1--6.

[9] PRESERVE Project, www.preserve-project.eu/Jun. 2015.

[10] W. Whyte, A. Weimerskirch, V. Kumar, and T. Hehn, "A Security Credential Management System for V2V Communications," in IEEE Vehicular Networking Conference (VNC), Boston, MA, Dec. 2013.

[11] P. Papadimitratos, "'On the road" - Reflections on the Security of Vehicular Communication Systems," in IEEE International Conference on Vehicular Electronics and Safety (ICVES), Columbus, OH, USA, Sep. 2008.

[12] M. Raya, D. Jungels, P. Papadimitratos, I. Aad, and J.-P. Hubaux, "Certificate Revocation in Vehicular Networks," Technical Report, EPFL, Switzerland, 2006

[13] L. Fischer, A. Aijaz, C. Eckert, and D. Vogt, "Secure revocable anonymous authenticated inter-vehicle communication (SRAAC)," in 4th Conference on Embedded Security in Cars (ESCAR). Berlin, Germany: Citeseer, Nov. 2006.

[14] F. Stumpf, L. Fischer, and C. Eckert, "Trust, Security and Privacy in VANETs - a Multilayered Security Architecture for C2CCommunication," in VDI/VW-Gemeinschaftstagung: Automotive Security, Wolfsburg, Germany, Nov. 2007, pp. 55--70.

[15] K. P. Laberteaux, J. J. Haas, and Y.-C. Hu, "Security Certificate Revocation List Distribution for VANET," in ACM VehiculAr InterNETworking, New York, NY, USA, Sep. 2008.

[16] J. J. Haas, Y.-C. Hu, and K. P. Laberteaux, "Design and Analysis of a Lightweight Certificate Revocation Mechanism for VANET," in Proceedings of the sixth ACM international workshop on VehiculAr InterNETworking, NY, USA, Sep. 2009.

[17] F. Schaub, Z. Ma, and F. Kargl, "Privacy Requirements in Vehicular Communication Systems," in International Conference on Computational Science and Engineering, 2009.

[18] J.-J. Haas, Y.-C. Hu, and K.-P. Laberteaux, "Efficient Certificate Revocation List Organization and Distribution," IEEE Journal on Selected Areas in Communications (JSAC), vol. 29, no. 3, pp. 595--604, 2011.

[19] P. Papadimitratos, G. Mezzour, and J.-P. Hubaux, "Certificate Revocation List Distribution in Vehicular Communication Systems," in ACM VANET, San Francisco, CA, Sep 2008.

[20] M. E. Nowatkowski and H. L. Owen, "Certificate Revocation List Distribution in VANETs Using Most Pieces Broadcast," in IEEE SoutheastCon, Concord, NC, USA, Mar. 2010.

[21] M. Nowatkowski, C. McManus, J. Wolfgang, and H. Owen, "Cooperative Certificate Revocation List Distribution Methods in VANETs," in International Ad Hoc Networks, Sep. 2009.

[22] "American Community Survey (ACS)," https://www.census.gov/ programs-surveys/acs/. Jun. 2015.

[23] "When, Where and How Much Motorists Drive," https://newsroom.aaa.com/2015/04/ new-study-reveals-much-motorists-drive/. Jun. 2016.

[24] J. Harding, G. Powell, R. Yoon, J. Fikentscher, C. Doyle, D. Sade, M. Lukuc, J. Simons, and J. Wang, "V2V Communications: Readiness of V2V Technology for Application," U.S. Department of Transportation - National Highway Traffic Safety Administration DOT HS 812 014, Tech. Rep., Aug. 2014.

[25] M. Raya, P. Papadimitratos, I. Aad, D. Jungels, and J.-P. Hubaux, "Eviction of Misbehaving and Faulty Nodes in Vehicular Networks," IEEE Journal on Selected Areas in Communications (JSAC), pp. 1557--1568, Oct. 2007.

[26] G. Rigazzi, A. Tassi, R. J. Piechocki, T. Tryfonas, and A. Nix, "Optimized Certificate Revocation List Distribution for Secure V2X Communications," IEEE Vehicular Technology Conference (VTC), Sep. 2017.

[27] M. Khodaei, H. Jin, and P. Papadimitratos, "Towards Deploying a Scalable \& Robust Vehicular Identity and Credential Management Infrastructure," in IEEE Vehicular Networking Conference (VNC), Paderborn, Germany, Dec. 2014.

[28] "Vehicle-to-Vehicle Communications Misbehavior Detection," Vehicle Safety Communications 6 (VSC6) Consortium Proprietary, 
Tech. Rep., Nov. 2018, available: https://wiki.campllc.org/display/ CPD/CAMP+Public+Documents+Home

[29] M. Harris, "Google Reports Self-driving Car Mistakes: 272 Failures and 13 Near Misses," https://www.theguardian.com/technology/ 2016/jan/12/google-selt-driving-cars-mistakes-data-reports Jan. 2016.

[30] M. Gerlach, A. Festag, T. Leinmüller, G. Goldacker, and C. Harsch, "Security Architecture for Vehicular Communication," in Workshop on Intelligent Transportation, Hamburg, Germany, Mar. 2007, pp. 119--124.

[31] E. N. Michael and L. O. Henry, "Scalable Certificate Revocation List Distribution in Vehicular Ad Hoc Networks," in IEEE GLOBECOM Workshops, Dec. 2010.

[32] P. Ardelean and P. Papadimitratos, "Implementation and Evaluation of Certificate Revocation List Distribution for Vehicular Ad-hoc Networks," EPFL, Tech. Rep., January 2009.

[33] H.-C. Hsiao, A. Studer, C. Chen, A. Perrig, F. Bai, B. Bellur and A. Iyer, "Flooding-Resilient Broadcast Authentication for VANETs," in ACM Mobile Computing and Networking, Las Vegas, Nevada, USA, Sep. 2011.

[34] V. T. Nguyen, J. Jose, X. Wu, and T. Richardson, "Secure Content Distribution in Vehicular Networks," arXiv e-prints, $\mathrm{p}$. arXiv:1601.06181, Jan. 2016.

[35] B. H. Bloom, "Space/Time Trade-offs in Hash Coding with Allowable Errors," Communications of the ACM, vol. 13, no. 7, pp. 422--426, July 1970.

[36] M. Mitzenmacher, "Compressed Bloom Filters," IEEE Transactions on Networking, vol. 10, no. 5, pp. 604--612, Dec. 2002.

[37] K. Rabieh, M. Pan, Z. Han, and V. Ford, "SRPV: A Scalable Revocation Scheme for Pseudonyms-Based Vehicular Ad Hoc Networks," in IEEE International Conference on Communications (ICC), Kansas City, MO, USA, May 2018, pp. 1--6.

[38] M. Khodaei, H. Noroozi, and P. Papadimitratos, "POSTER: Privacy Preservation through Uniformity," in Proceedings of the 11th ACM Conference on Security $\mathcal{E}$ Privacy in Wireless and Mobile Networks (ACM WiSec), Stockholm, Sweden, June 2018, pp. 279--280.

[39] C. Vaas, M. Khodaei, P. Papadimitratos, and I. Martinovic, "Nowhere to hide? Mix-Zones for Private Pseudonym Change using Chaff Vehicles," in IEEE Vehicular Networking Conference (VNC), Taipei, Taiwan, Dec. 2018.

[40] M. Khodaei and P. Papadimitratos, "Poster: Mix-Zones Everywhere: A Dynamic Cooperative Location Privacy Protection Scheme," in IEEE Vehicular Networking Conference (VNC), Taipei, Taiwan, Dec. 2018.

[41] -----, "Efficient, Scalable, and Resilient Vehicle-Centric Certificate Revocation List Distribution in VANETs," in Proceedings of the 11th ACM Conference on Security \& Privacy in Wireless and Mobile Networks (ACM WiSec), Stockholm, Sweden, June 2018.

[42] M. A. Simplicio Jr, E. L. Cominetti, H. K. Patil, J. E. Ricardini, and M. V. M. Silva, "ACPC: Efficient Revocation of Pseudonym Certificates using Activation Codes," Elsevier Ad Hoc Networks, July 2018.

[43] M. Khodaei, H. Jin, and P. Papadimitratos, "SECMACE: Scalable and Robust Identity and Credential Management Infrastructure in Vehicular Communication Systems," IEEE Transactions on Intelligent Transportation Systems, vol. 19, no. 5, pp. 1430--1444, May 2018.

[44] G. Marias, K. Papapanagiotou, and P. Georgiadis, "ADOPT: A Distributed OCSP for Trust Establishment in MANETs," in European Wireless Conference, Nicosia, Cyprus, April.

[45] C. Gañán, J. L. Muñoz, O. Esparza, J. Mata-Díaz, J. HernándezSerrano, and J. Alins, "COACH: Collaborative Certificate Status Checking Mechanism for VANETs," Network and Computer Applications, vol. 36, no. 5, Sep. 2013.

[46] V. Kumar, J. Petit, and W. Whyte, "Binary Hash Tree based Certificate Access Management for Connected Vehicles," in Proceedings of the 10th ACM Conference on Security \& Privacy in Wireless and Mobile Networks (ACM WiSec), Boston, USA, July 2017.

[47] M. A. S. Junior, E. L. Cominetti, H. K. Patil, J. Ricardini, L. Ferraz, and M. V. Silva, "Privacy-preserving Method for Temporarily Linking/Revoking Pseudonym Certificates in VANETs," in IEEE International Conference On Trust, Security And Privacy In Computing And Communications/12th IEEE International Conference On Big Data Science And Engineering (TrustCom/BigDataSE), New York, NY, USA, August 2018, pp. 1322--1329.

[48] M. A. Simplicio Jr, E. L. Cominetti, and H. K. Patil, "Privacypreserving Linkage/Revocation of VANET Certificates without LAs," https://eprint.iacr.org/2018/788.pdf. IEEE International
Conference on Trust, Security and Privacy in Computing and Communications, Aug. 2018.

[49] E. R. Verheul, "Activate Later Certificates for V2X-Combining ITS Efficiency with Privacy," IACR Cryptology ePrint Archive, vol. 2016, p. 1158, Dec. 2016.

[50] M. Khodaei and P. Papadimitratos, "The Key to Intelligent Transportation: Identity and Credential Management in Vehicular Communication Systems," IEEE Vehicular Technology Magazine, vol. 10, no. 4, pp. 63--69, Dec. 2015.

[51] N. Bißmeyer, "Misbehavior Detection and Attacker Identification in Vehicular Ad-Hoc Networks," Ph.D. dissertation, Technische Universität, Dec. 2014.

[52] M. Khodaei, "Secure Vehicular Communication Systems: Design and Implementation of a Vehicular PKI (VPKI)," Master's thesis, Division of Network and Systems Engineering, Royal Institute of Technology (KTH), Stockholm, Sweden, Oct. 2012.

[53] P. Papadimitratos, V. Gligor, and J.-P. Hubaux, "Securing Vehicular Communications-Assumptions, Requirements, and Principles," in ESCAR, Berlin, Germany, Nov. 2006, pp. 5--14.

[54] H. Noroozi, M. Khodaei, and P. Papadimitratos, "DEMO: VPKIaaS: A Highly-Available and Dynamically-Scalable Vehicular PublicKey Infrastructure," in Proceedings of the 11th ACM Conference on Security $\mathcal{E}$ Privacy in Wireless and Mobile Networks (ACM WiSec), Stockholm, Sweden, June 2018, pp. 302--304.

[55] M. Khodaei, H. Noroozi, and P. Papadimitratos, "Scaling Pseudonymous Authentication for Large Mobile Systems," in Proceedings of the 12th ACM Conference on Security \& Privacy in Wireless and Mobile Networks (ACM WiSec), Miami, FL, USA, May 2019.

[56] M. Khodaei and P. Papadimitratos, "Evaluating On-demand Pseudonym Acquisition Policies in Vehicular Communication Systems," in Proceedings of the First International Workshop on Internet of Vehicles and Vehicles of Internet (IoV/VoI), Paderborn, Germany, July 2016.

[57] M. Khodaei, A. Messing, and P. Papadimitratos, "RHyTHM: A Randomized Hybrid Scheme To Hide in the Mobile Crowd," in IEEE Vehicular Networking Conference (VNC), Torino, Italy, Nov. 2017.

[58] P. T. Eugster, P. A. Felber, R. Guerraoui, and A.-M. Kermarrec, "The Many Faces of Publish/Subscribe," ACM computing surveys, vol. 35, no. 2, pp. 114--131, June 2003.

[59] Y. Huang and H. Garcia-Molina, "Publish/Subscribe in a Mobile Environment," Wireless Networks, vol. 10, no. 6, pp. 643--652, Nov. 2004.

[60] A. Perrig, R. Canetti, J. D. Tygar, and D. Song, "The TESLA Broadcast Authentication Protocol," RSA Cryptobytes, vol. 5, no. 2, pp. 2--13, August 2002.

[61] S. Das, A. Nandan, and G. Pau, "SPAWN: A Swarming Protocol for Vehicular Ad-Hoc Wireless Networks," in ACM workshop on VANET, Philadelphia, PA, USA, Oct. 2004.

[62] 3GPP-TR-36.885, "3rd Generation Partnership Project; Technical Specification Group Radio Access Network; Study on LTE-based V2X Services; (Release 14)," https://www.tech-invite.com/3m36/ tinv-3gpp-36-885.html. June 2016.

[63] Q. Dang, Recommendation for Applications using Approved Hash Algorithms. US Department of Commerce, National Institute of Standards and Technology, August 2015.

[64] T. Gerbet, A. Kumar, and C. Lauradoux, "The Power of Evil Choices in Bloom Filters," in IEEE/IFIP International Conference on Dependable Systems and Networks (IFIP DSN), Rio de Janeiro, Brazil, June 2015, pp. 101--112.

[65] A. Broder and M. Mitzenmacher, "Network Applications of Bloom Filters: A Survey," Internet mathematics, vol. 1, no. 4, pp. 485--509, January 2004.

[66] "Bitmain Antminer S9 Review," https://www. buybitcoinworldwide.com/mining/hardware/antminer-s9/. Nov. 2017.

[67] "Antpool, advanced bitcoin mining pool," https://v3.antpool.com/ home, Nov. 2017.

[68] G. Calandriello, P. Papadimitratos, J.-P. Hubaux, and A. Lioy, "On the Performance of Secure Vehicular Communication Systems," IEEE TDSC, vol. 8, no. 6, pp. 898--912, Nov. 2011.

[69] "Omnet++," https://www.omnetpp.org/. Jun. 2017.

[70] M. Behrisch, L. Bieker, J. Erdmann, and D. Krajzewicz, "SUMO - Simulation of Urban MObility: An Overview," in The 3rd International Conference on Advances in System Simulation, Barcelona, Spain, Oct. 2011, pp. 55--60. 
[71] L. Codeca, R. Frank, and T. Engel, "Luxembourg Sumo Traffic (LuST) Scenario: 24 Hours of Mobility for Vehicular Networking Research," in IEEE Vehicular Networking Conference (VNC), Kyoto, Japan, Dec. 2015.

[72] "IEEE Standard for Wireless Access in Vehicular Environments (WAVE) - Networking Services," IEEE Vehicular Technology Society - Standards Association, Tech. Rep., Jan. 2016.

[73] "Scalable Bloom Filter Implemented in Python," https://github. com/jaybaird/python-bloomfilter/. Mar. 2013.

[74] Y. Liang, H. Liu, and D. Rajan, "Optimal Placement and Configuration of Roadside Units in Vehicular Networks," in IEEE Vehicular Technology Conference (VTC), Yokohama, Japan, May 2012.

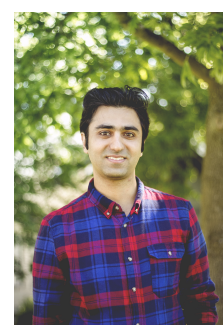

Mohammad Khodaei earned his diploma in software engineering from Azad University of Najafabad in Isfahan, Iran, in 2006 and his M.S. degree in information and communication systems security from KTH Royal Institute of Technology, Stockholm, Sweden, in 2012. He is currently pursuing his Ph.D. degree at the Networked Systems Security Group, KTH, under the supervision of Prof. Panos Papadimitratos. His research interests include security and privacy in VANETs, smart cities, and the Internet of Things.

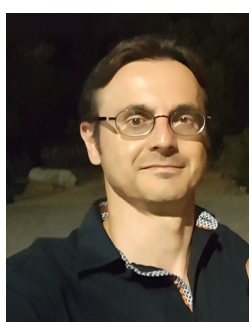

Panos Papadimitratos earned his Ph.D. degree from Cornell University, Ithaca, NY. At KTH, Stockholm, Sweden, he leads the Networked Systems Security lab, and he is a member of the steering committee of the Security Link center. He has delivered numerous invited talks, keynotes, panel addresses, and tutorials in flagship conferences. He serves or served as: Associate Editor of the IEEE TMC and the ACM/IEEE TON journals; member of the PETS Editorial and Advisory Boards, and the ACM WiSec and CANS conference steering committees; program chair for the ACM WiSec'16, TRUST'16, CANS'18 conferences; general chair for ACM WISec'18, PETS'19, and IEEE EuroS\&P'19. He is a Fellow of the Young Academy of Europe, a Knut and Alice Wallenberg Academy Fellow, and IEEE Fellow. His group web-page is: https://www.eecs.kth.se/nss 